\title{
Co-expressed genes prepositioned in spatial neighborhoods stochastically associate with SC35 speckles and RNA polymerase II factories
}

\author{
Dietmar Rieder • Christian Ploner • Anne M. Krogsdam • Gernot Stocker • \\ Maria Fischer · Marcel Scheideler · Christian Dani · Ez-Zoubir Amri · \\ Waltraud G. Müller · James G. McNally $\cdot$ Zlatko Trajanoski
}

Received: 8 May 2013 / Revised: 2 August 2013 / Accepted: 28 August 2013 / Published online: 12 September 2013

(C) Springer Basel 2013

\begin{abstract}
Chromosomally separated, co-expressed genes can be in spatial proximity, but there is still debate about how this nuclear organization is achieved. Proposed mechanisms include global genome organization, preferential positioning of chromosome territories, or gene-gene sharing of various nuclear bodies. To investigate this question, we selected a set of genes that were co-expressed upon differentiation of human multipotent stem cells. We applied a novel multi-dimensional analysis procedure which revealed that prior to gene expression, the relative position of these genes was conserved in nuclei. Upon stem cell
\end{abstract}

Electronic supplementary material The online version of this article (doi:10.1007/s00018-013-1465-3) contains supplementary material, which is available to authorized users.

D. Rieder · A. M. Krogsdam · G. Stocker · M. Fischer ·

Z. Trajanoski $(\square)$

Division of Bioinformatics, Biocenter, Innsbruck Medical

University, Innrain 80, 6020 Innsbruck, Austria

e-mail: zlatko.trajanoski@i-med.ac.at

C. Ploner

Department of Plastic, Reconstructive and Aesthetic Surgery, Innsbruck Medical University, 6020 Innsbruck, Austria

M. Scheideler

Institute for Genomics and Bioinformatics, Graz University

of Technology, 8010 Graz, Austria

C. Dani $\cdot$ E.-Z. Amri

Faculty of Medicine, Institute of Developmental Biology and Cancer, CNRS, University of Nice Sophia-Antipolis, Nice, France

W. G. Müller · J. G. McNally

Laboratory of Receptor Biology and Gene Expression, National Cancer Institute, National Institutes of Health, Bethesda,

MD, USA differentiation and concomitant gene expression, we found that co-expressed genes were closer together. In addition, we found that genes in the same $1-\mu \mathrm{m}$-diameter neighborhood associated with either the same splicing speckle or to a lesser extent with the same transcription factory. Dispersal of speckles by overexpression of the serine-arginine (SR) protein kinase cdc2-like kinase Clk2 led to a significant drop in the number of genes in shared neighborhoods. We demonstrate quantitatively that the frequencies of speckle and factory sharing can be explained by assuming stochastic selection of a nuclear body within a restricted sub-volume defined by the original global gene positioning present prior to gene expression. We conclude that the spatial organization of these genes is a two-step process in which transcription-induced association with nuclear bodies enhances and refines a pre-existing global organization.

Keywords Nuclear organization $\cdot$ Spatial gene positioning $\cdot$ Splicing speckles $\cdot$ Transcription factories

$\begin{array}{ll}\text { Abbreviations } \\ \text { BAC } & \text { Bacterial artificial chromosome } \\ \text { CT } & \text { Chromosome territory } \\ \text { hMADS } & \begin{array}{l}\text { Human multipotent adipose tissue-derived } \\ \text { stem cells }\end{array}\end{array}$

\section{Introduction}

It is now clear that the cell nucleus is spatially organized. For example, specific genes are found more frequently in certain radial positions or at certain nuclear hallmarks, and specific chromosomes have a tendency to be found near to each other and to be positioned more towards either the nuclear interior or periphery. In all cases, this organization 
varies among different cell types or tissues and appears to be probabilistic: the structures of interest are located near to each other at rates well above chance but far from $100 \%$ of the time. How this probabilistic organization arises remains unclear.

One particularly intriguing form of nuclear organization is the tendency for co-expressed genes to be located in a nuclear neighborhood. This can be accomplished either by arranging genes on the linear sequence of a chromosome or by positioning otherwise-separated loci near each other in the nuclear space. In the latter case, this gene positioning is not absolute, but as found in single cell analyses it arises at frequencies in the range of about $1 \%$ to more than $30 \%$ and occurs at distances $<1 \mu \mathrm{m}[1-4]$.

Positioning co-expressed genes next to each other on the linear sequence of the chromosome is often seen in Prokaryotes where co-regulated genes are arranged in "operons". A similar organization was observed in Saccharomyces cerevisiae [5], Caenorhabditis elegans [6], mouse [7], and other higher Eukaryotes [8-11]. Moreover, spatial partitioning of chromosomes into ( 100-kb-megabase-sized) topological domains is emerging as a basic feature of the regulatory architecture of eukaryotic genomes [12-14].

There is also evidence for non-random positioning of distant genes, located Mbps apart or on different chromosomes. Single-cell analyses revealed spatial positioning at close nuclear locations in the sub-micrometer range for active $[1-4,15]$ as well as for inactive genes [16, 17]. Moreover, the use of genome-wide chromosome conformation capture (3C)-based assays, such as 4C, e4C, or ChIAPET, interrogating large populations of cells, revealed many potential interactions of active and inactive genes in different cell types [4, 18-21]. However, even within cells of the same type, i.e., erythroid cells, there are different conclusions about how close spatial positioning of otherwise distant genes might occur [1-4].

One model is based on SC35 splicing speckles [2, 3, 22, 23]. Frequent sharing of the same splicing speckle has been demonstrated for $\alpha$ - and $\beta$-globin and other erythroid-specific genes in human erythroblast cells [2,3] as well as for key adipogenic genes in porcine adipocytes [23].

An alternative model for spatial organization is based on shared RNA polymerase II organized in "factories". These so-called "transcription factories" are discrete sites in the nucleus composed of 4-30 RNA polymerase molecules, and are associated with many other molecules involved in transcriptional activation and mRNA processing. Several hundreds to thousands of these sites exist in eukaryotic nuclei and at each of them multiple different genes may be transcribed at the same time [24-28]. Examples supporting this model are the Myc and Igh genes in mouse B lymphocytes, which are located on different chromosomes [15] or the $H b a$ or $H b b$ genes in mouse erythroid cells, which are positioned together with other erythroid genes at shared polymerase factories despite the fact that the loci are on different chromosomes [4].

A third model that has been considered is that spatial positioning of genes could arise from a more global organization of the genome within the nucleus. It is known that some chromosomes tend to be near each other [7, 29, 30], and that specific genes may generally be positioned at specific locations within the nucleus [7, 31, 32].

These three different models operate on potentially different spatial scales. Genes associated with the same nuclear body should be in shared neighborhoods comparable to the size of the body, namely $\sim 40-198 \mathrm{~nm}$ for a transcription factory and $\sim 0.5-2 \mu \mathrm{m}$ for a speckle. Higherorder levels of organization based on chromosome folding and relative chromosome positioning are expected to operate on a spatial scale of at least several microns.

In many previous studies, only one of the preceding mechanisms for positioning of genes into nuclear neighborhoods has been investigated, making it uncertain to what extent other mechanisms might also contribute. In this study, we systematically explore the spatial organization of seven co-expressed genes in human multipotent adiposederived stem (hMADS) cells [33] and by considering all of the three preceding models we ask how the positioning of genes might arise. We predict that merely adjacent positioning of chromosome territories cannot conclusively explain our observations on gene positioning in spatial neighborhoods. Rather, we find that close positioning of active genes can be explained by random association with nearby SC35-enriched splicing speckles and RNA polymerase II factories, but within restricted nuclear domains to which genes must be prepositioned based on a conserved higher spatial organization of the genome. The frequency of association with factories or speckles is determined by the relative number of these two types of nuclear bodies present within the restricted sub-volume. Thus, we propose a model that integrates all three alternative explanations for gene positioning described above, and that can also potentially account for observations made on different genes in different systems.

\section{Results}

Non-random spatial topology prepositions gene in the nucleus

A bioinformatic analysis of gene expression microarray data of adipogenesis in hMDAS cells [34] revealed 12 groups of coordinately expressed genes (supplemental information Fig. S1). We chose seven genes (C4orf18, GLUL, SAMHD1, AGTR1, ANGPTL1, FKBP5, SPON2; 
see also Table 1) from one of these groups and asked if they are spatially organized by specific positioning. We used 3D mFISH to simultaneously localize all seven genes in $\sim 400$ nuclei of preconfluent (80-90\% confluence) and induced hMADS cells (Fig. 1a). We reasoned that the topology of these genes in the nucleus is reflected by their relative distances to each other. Therefore it should be possible to compare different nuclei by comparing these distances. We measured the center-to-center distance between each pair of genes and since the two alleles of a gene are indistinguishable by DNA-FISH, we considered only the shorter distance between the two alleles of a given gene pair. These distances were normalized by the nuclear volume and for each nucleus a vector of normalized distances was created. We then calculated the pairwise Pearson correlation coefficients between these vectors and visualized them as heatmaps.

The upper triangles in Fig. 1b-e illustrate such heatmaps of significant $(p<0.05)$ correlations. Each square represents the color-coded $-\log _{10} p$ value of the corresponding pairwise correlation. Surprisingly, the spatial organization of the seven genes was significantly correlated in a high number of nuclei. We observed this in preconfluent (Fig. 1b) as well as in induced cells (Fig. 1c). Moreover, it appeared that this organization is preserved not only among nuclei in the same physiological condition but also between nuclei in the induced and uninduced states (Fig. 1d).

To eliminate the possibility that this high correlation could be obtained by chance, we randomly permuted the measured nuclear gene-gene distances within each vector and recalculated the correlation coefficients and $p$ values. The lower triangles in Fig. 1 demonstrate that the correlation of vectors with the correct distance assignment could not be produced by an arbitrary distance assignment. Moreover, entirely random gene-gene distances $(0 \leq d \leq$ mean

Table 1 Genomic locations and BAC clones

\begin{tabular}{llll}
\hline Gene & Chr\# & Location Mbps & BAC-clone \\
\hline C4orf18 & 4 & 159.3 & RP11-89C4 \\
GLUL & 1 & 180.6 & RP1-223H12 \\
SAMHD1 & 20 & 35.0 & RP1-132F21 \\
AGTR1 & 3 & 149.9 & RP11-505J9 \\
ANGPTL1 & 1 & 177.1 & RP4-595C2 \\
FKBP5 & 6 & 35.7 & RP3-368C2 \\
SPON2 & 4 & 1.1 & RP11-20I20 \\
ANXA6 & 5 & 150.5 & RP11-507E2 \\
COL6A1 & 21 & 47.4 & RP11-640F21 \\
CR & 7 & $10.7-10.9$ & RP11-466C11 \\
\hline
\end{tabular}

Chromosomal coordinates and corresponding BAC clones for generating probes of genes studied by DNA-FISH: chromosomal locations are shown in megabase pairs. $C R$ is a genomic control region that has no annotated protein-coding gene diameter of hMADS nuclei) for seven simulated gene pairs, could also not reproduce the observed correlation (Fig. 1e).

Two of the studied genes, GLUL and ANGPTL1, are only separated by $3.5 \mathrm{Mbps}$ on chromosome 1 (see Table 1), and therefore they are likely to be located at similar spatial distance in any cell under any condition. Thus, this will naturally increase the correlation and therefore we repeated the analysis by excluding one of the partners $(G L U L)$. However, the spatial organization of the remaining six genes, was again significantly correlated in remarkable number of nuclei and was clearly different from random organization (supplemental information Fig. S2 a-c). In addition to $G L U L$, we also excluded $C 4$ orf 18 , which is located $>158$ Mbps away from SPON2 on chromosome 4 (see Table 1), but still the correlation was not strongly affected and indicated a non-random nuclear topology (supplemental information Fig. S2 d-f).

These results suggest that a higher-order and non-random spatial gene organization prepositions genes relative to each other into certain nuclear sub-volumes. This relative positioning is preserved between different nuclei and does not undergo dramatic changes upon induction of differentiation.

Coordinately expressed genes are frequently located in shared neighborhoods and this occurs more often with transcriptional upregulation

To further explore this nuclear organization, we next asked if there were any pairs of genes which tended to be in a shared sub-volume or "neighborhood". Although five of the seven genes we examined were coordinately expressed, they were not near each other on the same chromosomes, so we would not expect to find these genes in spatial proximity within the nucleus based solely on their linear positions within the chromosomes. We scored genes as occupying a shared neighborhood if they were within a distance of $1 \mu \mathrm{m}$. This threshold should account for organization mediated by most nuclear bodies, which have a maximal diameter of $\sim 1 \mu \mathrm{m}$, like transcription factories [28], splicing speckles [35], paraspeckles [36], PML bodies [37], or Cajal bodies [38].

We first analyzed induced cells, and found several gene pairs positioned in shared neighborhoods at unexpectedly high frequencies, namely: FKBP5 and SPON2 in $17.2 \%$, C4orf18 and SPON2 in $13 \%$, C4orf18 and FKBP5 in $12.8 \%$, and finally GLUL and ANGPTL1 in $68 \%$ (Fig. 2a, supplemental information Table 2, Fig. S6 a-h). Typically, only one of the two alleles showed association with its partner, but all of these frequencies are much more than would be expected by pure chance $(\sim 0.55 \%$ see below). Moreover, many of them are higher than determined by actual measurements of a pair of functionally unrelated 

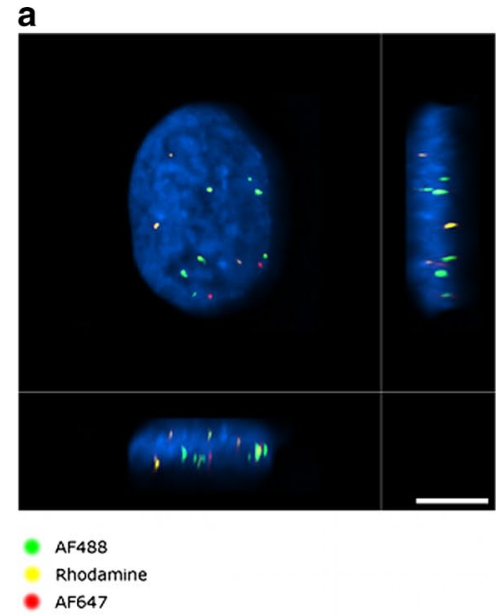

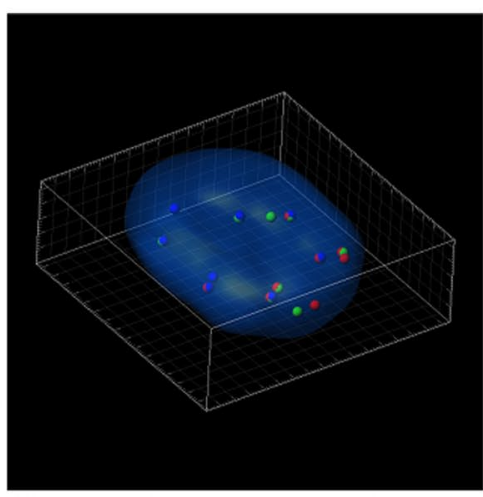

$$
\begin{aligned}
& \text { - AF488 } \\
& \text { - Rhodamine } \\
& \text { - AF647 }
\end{aligned}
$$

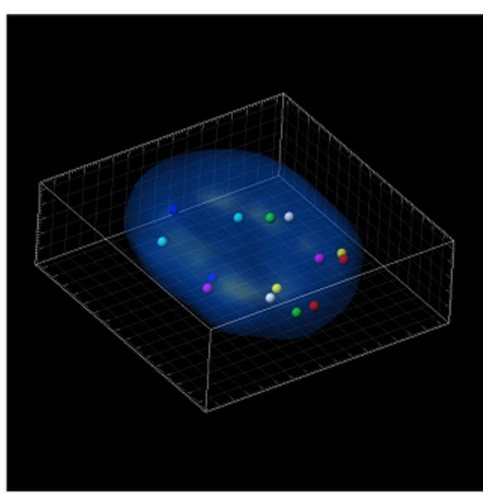

C4Orf18

- glul.

- SAMHD1

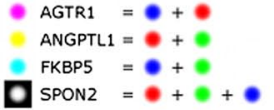

- AGTR1 $=\bullet+\bullet$

- SPON2 $=+++$

b nucleus/nucleus correleation preconfluent: $-\log _{10} p \quad$ C
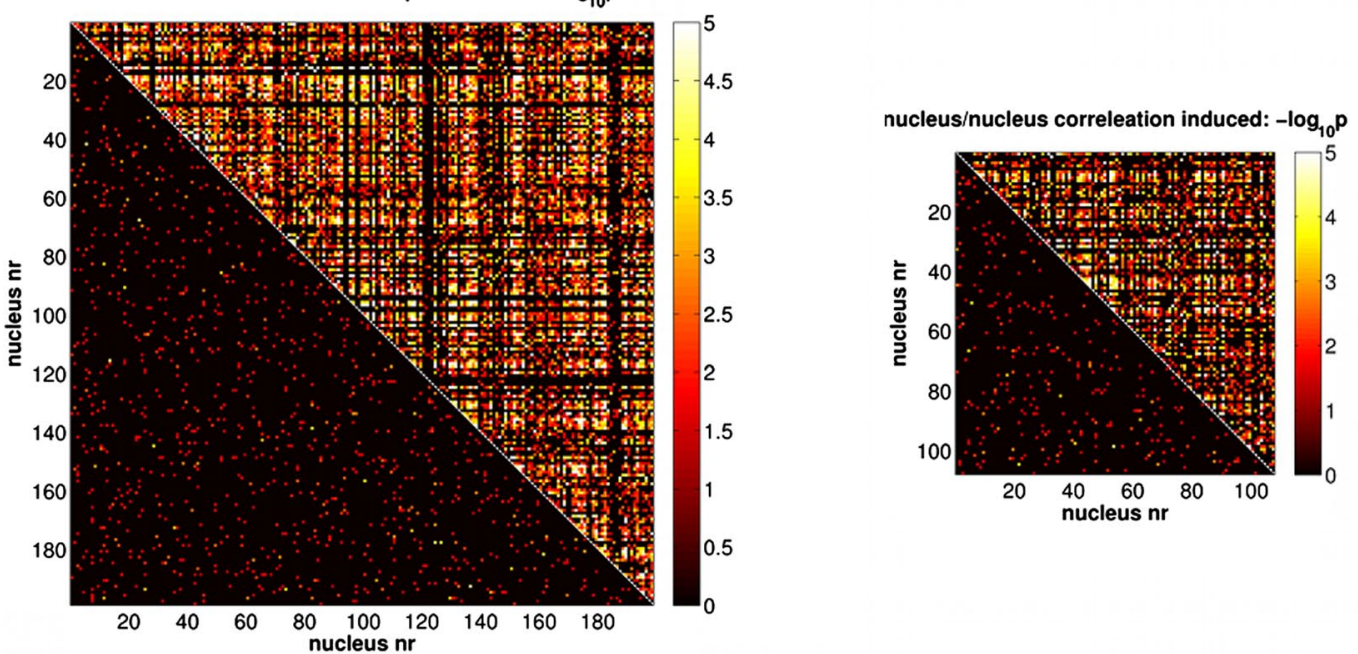

d
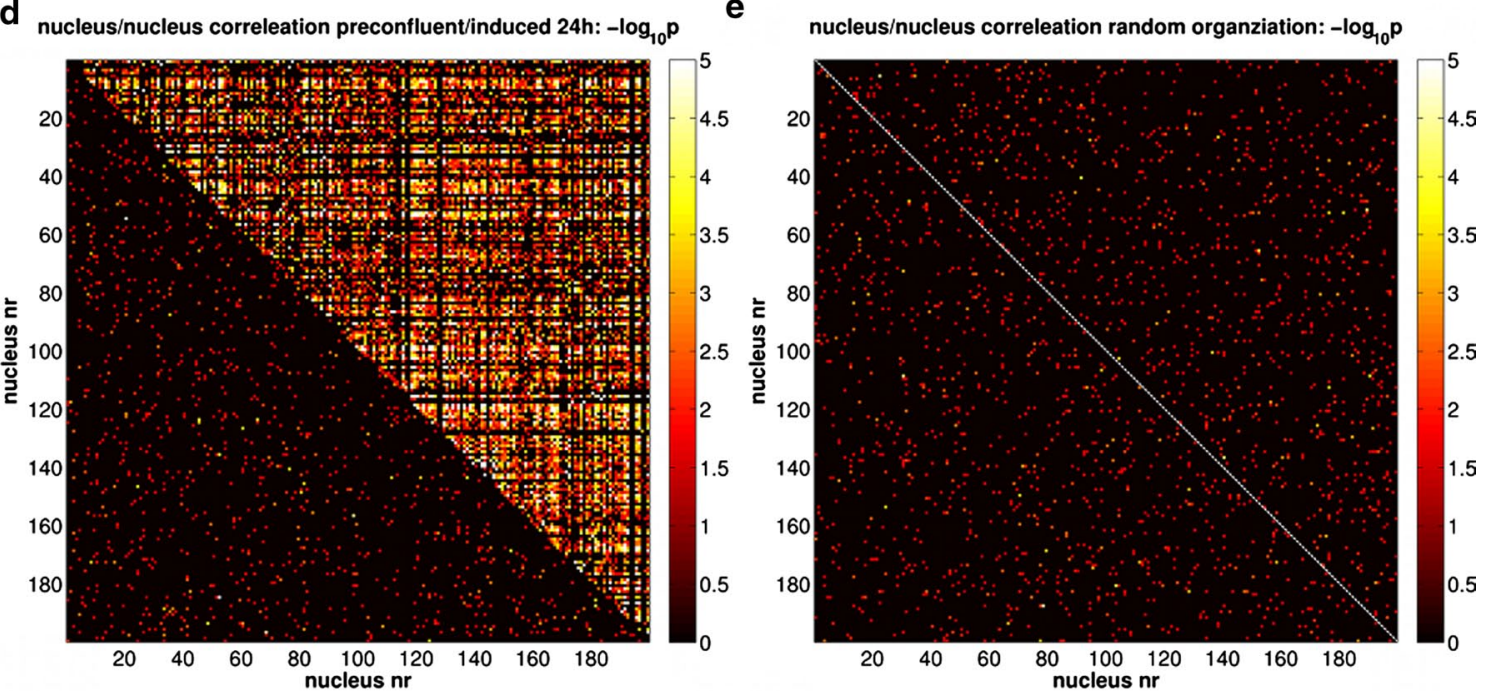

housekeeping genes (ANXA6/COL6A1: $3.2 \% n=125)$ or as measured for the two housekeeping genes and a genomic control region that has no annotated protein coding genes
$($ ANXA6/CR: $2.8 \% n=106$, COL6A1/CR: $1.9 \% n=106)$ (see also Table 1, supplemental information Tables 5-7). We then repeated the gene-pair analyses using uninduced 
४Fig. 1 Spatial positioning of coordinately expressed genes (a). Left maximum intensity projections (xy, $\mathrm{xz}, \mathrm{yz}$ ) of DNA mFISH in hMADS cells are shown. Seven different genes were labeled using all possible combinations of three fluorophores, namely Alexa Fluor 488 (green), Rhodamine (orange) and Alexa Fluor 647 (purple)—scale bar $=5 \mu \mathrm{m}$. Middle signals of the individual fluorophores as detected by a spot detection algorithm. Right identification of the seven single genes by decoding the color code. b-e Correlation analysis of nuclear organization. Distance vectors were used to describe the spatial organization of seven coordinately expressed genes in each nucleus. Each pixel of the shown heatmaps represents a color-coded $-\log _{10}(p)$ value $(p<0.05)$ of the correlation between two single nuclei. To the right of each heatmap, the color-coded scale for the $-\log _{10}(p)$ values is shown. The upper triangles of the heatmaps display significant correlations between each pair of nuclei in 200 preconfluent cells (b), 110 induced cells (c), and in a comparison of both (d). The lower triangles show the results of a random permutation test. e Simulation of a random positioning model could not reproduce the observed topology correlations in hMADS cells (see text for details)

cells and compared the results (Fig. 2b, supplemental information Table 3) with data obtained from induced cells (Fig. 2a, supplemental information Table 2). Interestingly, there was a significant difference in frequency of close positioning between the two developmental states for two gene pairs. When cells changed from the uninduced to the induced state, we observed an increase from 1.8 to $12.8 \%$ (Fisher's exact test, $p<10^{-5}$ ) for C4orf18/FKBP5 and an increase from 8.2 to $17.2 \%$ (Fisher's exact test, $p<6 \times 10^{-3}$ ) for FKBP5/SPON2 (supplemental information Table 4). This increase was not due to a reduction in nuclear size upon induction since we could not detect a significant difference in nuclear volume between induced and uninduced cells. Rather, this increase is more likely connected to the significant increase in the transcriptional activity of the three genes as measured by quantitative RTPCR (C4orf18 and SPON2 2-8 fold, FKBP5 40 fold, Fig. 2c). In general, our quantification of absolute levels of expression revealed that six out of seven genes showed a low basal expression in preconfluent hMADS and were then strongly upregulated by hormonal induction, whereas SPON2 was already highly active in the uninduced state (Fig. 2d).

We further verified the impact of transcriptional activity on neighborhood sharing by blocking transcription with 5,6-dichlorobenzimidazole 1- $\beta$-D-ribofuranoside (DRB), which is known to inhibit transcriptional elongation [3941]. When we treated induced hMADS cells for $1 \mathrm{~h}$ with $50 \mu \mathrm{g} / \mathrm{ml}$ DRB and performed dual-color DNA-FISH using probes for FKBP5 and SPON2, we detected a significant reduction in neighborhood sharing, which dropped from 17.2 to $7.6 \%$ (Fisher's exact test $p<0.005, n=185$ ). The neighborhood sharing frequency of FKBP5/SPON2 in DRB-treated preconfluent hMADS cells dropped only moderately from 8.3 to $5.1 \%$ (Fisher's exact test $p>0.23$, $n=177$ ), which we assume is due to the already very low expression of FKBP5 in uninduced hMADS cells (see Fig. 2d). It is important to note that DRB treatment did not irreversibly damage gene transcription in hMADS cells, as nascent transcription recovered in DRB-treated cells after a 45-min incubation time in normal growth medium (see supplemental information Fig. S7).

To test if there is a general tendency for closer gene positioning in induced cells, we counted all pairs of genes within $1 \mu \mathrm{m}$-diameter neighborhoods in both states and compared the two distributions (Fig. 2e). We found that there was a significant ( $t$ test, $p<0.006$ ) shift towards a higher frequency of close gene-gene positioning in the induced state. The same could be observed when we extended the analysis from gene pairs to gene triplets (Fig. 2f). Finally, we verified that the genes within shared neighborhoods were transcriptionally active, by application of dual-color RNA-FISH experiments using intron probes for FKBP5 and SPON2 (Fig. 3).

Taken together, these results show that the seven coordinately expressed genes-chosen only based on their expression profile-can share the same neighborhoods and that the frequency at which this positioning occurs can increase upon induction of differentiation and concomitant transcriptional up-regulation. We observed this for pairs which either lie on different chromosomes or in the case of C4orf18 and SPON2 reside on the same chromosome but are widely separated ( $>158 \mathrm{Mbps}$ ). As expected, we also observed neighborhood sharing for two of the genes, GLUL and ANGPTL1, which are closely located on chromosome 1. Thus, we next investigated how spatial positioning of widely spaced genes on different chromosomes might arise.

Frequent close chromosome territory location is not sufficient to explain positioning of specific gene pairs in shared neighborhoods

Chromosome territories (CTs) can be preferentially positioned near each other [7, 30], and furthermore intermingling of neighboring territories can occur more [42] or less $[43,44]$ extensively. Thus, it is possible that the observed frequency at which specific gene pairs were located in shared neighborhoods could simply reflect a high frequency of close territory positioning and intermingling.

Therefore we examined the chromosome territory locations of three gene pairs: \#1-SPON2 and FKBP5; \#2GLUL and SPON2; \#3-GLUL and FKBP5. Gene pair \#1 showed relatively high spatial association (17.2\%), while gene pairs \#2 and \#3 showed relatively low association (1.8 and $2.4 \%$ ) (Fig. 2a, b). We co-hybridized specific whole chromosome painting probes for chromosome 1, 4, and 6 to preserved nuclei and recorded $>803 \mathrm{D}$-stacks of uninduced and induced hMADS cells (Fig. 4a). 


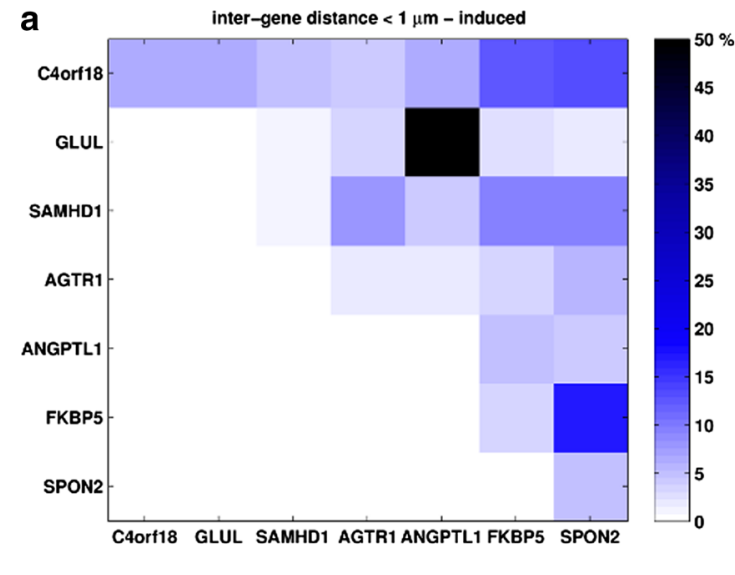

C

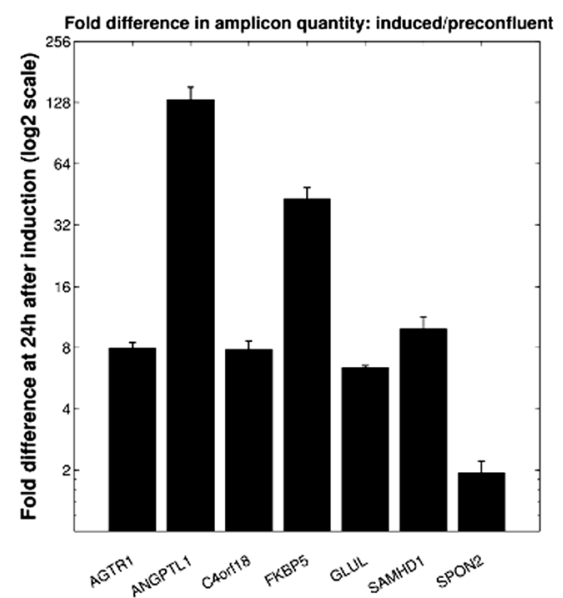

e

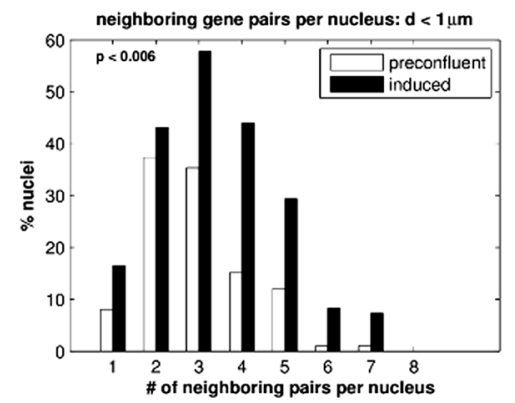

Fig. 2 Coordinately expressed genes in spatial neighborhood. The center-to-center distance between each gene pair of these seven genes was measured in $\sim 200$ nuclei of induced (a) and $\sim 200$ preconfluent (b) cells. The heatmaps represent color-coded percentages of gene pairs found to be separated by a spatial distance of $<1 \mu \mathrm{m}$. These pairs were then scored as positioned in spatial neighborhood. c, d RT-qPCR analysis of AGTR1, ANGPTL1, C4orf18, FKBP5, GLUL, SAMHD1, and SPON2 in hMADS cells (three biological replicates, error bars SE of means). $\mathbf{c}$ Fold changes between preconfluent and induced cells (24-h induction), $y$-axis in $\log 2$ scale. d Absolute expression levels in

We found that a considerable fraction $(27.9 \%)$ of the inspected CT pairs (CT 4/6) of gene pair \#1 were located directly adjacent and touched or partially overlapped each other (CT distance $\leq 0 \mu \mathrm{m}$, Fig. $4 \mathrm{~b}$ ). However, we obtained

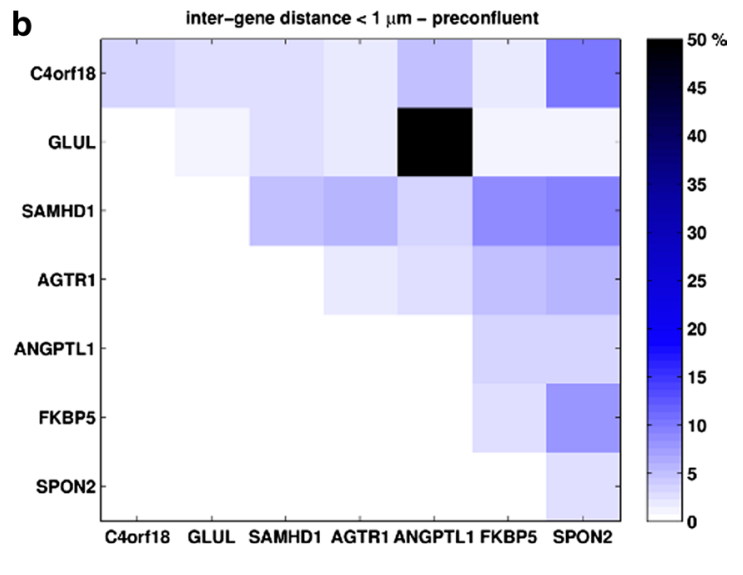

d

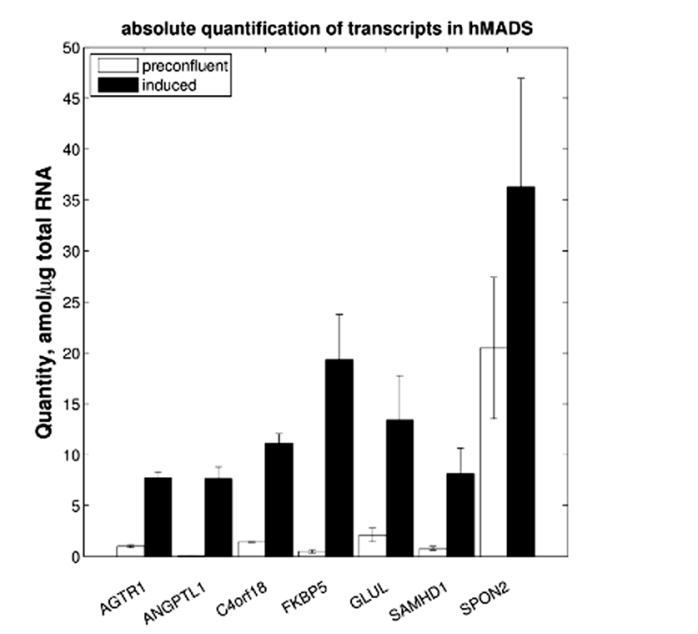

f

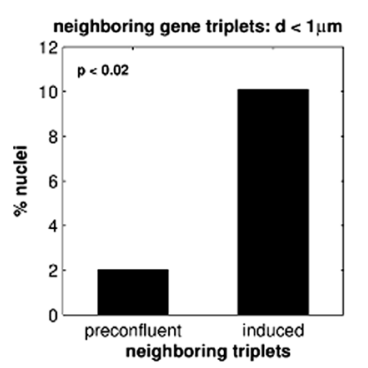

amol per $\mu \mathrm{g}$ total RNA as measured in preconfluent and induced cells (24-h induction). e Distribution of spatially neighboring gene pairs counted in nuclei of induced (black) and uninduced (white) cells. In induced cells, the number of gene pairs in spatial neighborhood per nucleus is significantly $(p<0.006)$ higher as compared to the number in uninduced cells. $\mathbf{f}$ Number of neighboring gene triplets in induced and preconfluent cells. A gene triplet is defined as a group of three genes in close proximity where the distance between any pair is $<1 \mu \mathrm{m}$

very similar numbers when we measured the distances of the CT pairs of gene pair \#2 and \#3 (CT 4/1-29.1\%, CT $1 / 6-31.3 \%)$. These three spatial distributions did not significantly differ ( $t$ test, $p>0.05$ ). This suggests that close 
Table 2 Measured association rates of SPON2 and FKBP5 at nuclear bodies

\begin{tabular}{lll}
\hline & SC35 speckle & $\begin{array}{l}\text { RNA polymerase } \\
\text { II factory }\end{array}$ \\
\hline fa (SPON2) & $99.0 \%$ & $90.5 \%$ \\
$\mathrm{fb}($ FKBP5) & $51.0 \%$ & $93.5 \%$ \\
$\mathrm{~N}_{\text {tot }}$ (\# in entire nucleus) & $32 \pm 6$ & $403 \pm 61$ \\
$\mathrm{~N}_{\text {Ishell }}$ (\# in inner four shells) & $20 \pm 4$ & $170 \pm 21$ \\
\hline
\end{tabular}

$\mathrm{fa}$ and $\mathrm{fb}$ are the independent association frequencies of SPON2 and FKBP5 at either SC35 speckles or RNA polymerase II factories. We took $\mathrm{fa}, \mathrm{fb}$, and the observed number of speckles or factories $\left(\mathrm{N}_{t o t}\right)$ to predict co-association rate (fe, see Table 2) of the two genes at these bodies by using the formula fe $=1 / N_{\text {tot }} \times 1 / f_{\mathrm{a}} \times 1 / f_{\mathrm{b}} \times 4$. For the prediction of co-association in the inner four nuclear shells we used the number of bodies $\left(\mathrm{N}_{\text {Ishell }}\right)$ in that restricted sub-volume

Table 3 Co-association rates of SPON2 and FKBP5 at nuclear bodies

\begin{tabular}{lll}
\hline & SC35 speckle & $\begin{array}{l}\text { RNA polymerase } \\
\text { II factory }\end{array}$ \\
\hline $\begin{array}{l}\text { Observed (\%) } \\
\begin{array}{l}\text { Predicted for entire nuclear } \\
\text { volume (\%) }\end{array}\end{array}$ & 12.0 & 3.0 \\
$\begin{array}{l}\text { Predicted for inner four } \\
\text { nuclear shells }\end{array}$ & $9.8 \pm 1.9 \%$ & $0.8 \pm 0.1$ \\
\hline
\end{tabular}

Observed and predicted co-association rates of SPON2 and FKBP5 at either SC35 speckles or RNA polymerase II factories. The predictions for co-association in the entire nuclear volume and in the inner four nuclear shells are based on the measured values shown in Table 1

CT positioning alone is not sufficient to explain the more frequent close positioning of the genes in pair \#1 in comparison to the genes in pairs \#2 and \#3. Rather, it indicates that there exists an additional higher-order organization and/or additional factors which specifically situate genes in a more confined sub-volume.

Genes in shared neighborhoods frequently associate with the same SC35 splicing speckles

An alternative explanation for close positioning of coordinately expressed genes has come from a study in erythroid cells, which showed that active erythroid genes are frequently localized at SC35-enriched speckles in human intermediate erythroblasts $[2,3]$. To test if speckle association was present in the hMADS cells, we performed triplecolor RNA immuno-FISH and used an antibody against SC35 and intron probes for FKBP5 and SPON2. We found that $68 \%$ of the FKBP5 and SPON2 RNA-FISH signals that were separated by less than $1 \mu \mathrm{m}$ were also contacting the same SC35 speckle (Fig. $5 b)(n=121)$. This means that of the $17.2 \%$ of nuclei that showed close positioning of these two genes, $68 \%$ of these spatial associations arose from association of the two genes with a shared splicing speckle. Thus, splicing speckle association can account for submicron association of these two genes in $0.68 \times 17.2=\sim 12 \%$ of total nuclei.

A model with completely random gene-pair/SC35 contacts is not sufficient to explain the observed speckle co-associations

We first considered a random model to explain the SC35 speckle co-association frequencies that we observed for the gene pair FKBP5/SPON2. Speckles are quite large in size $(0.5-2 \mu \mathrm{m})$ and therefore they occupy a large volume in the nucleus, increasing the odds that two genes would encounter the same speckle by chance. We tested this possibility in a simulation by randomly placing two genes (diameter $320 \mathrm{~nm}$ ) into 3D-reconstructed nuclei derived from images obtained by immunofluorescence staining of SC35. We scored the genes as neighboring if their center-to-center distance was $<1 \mu \mathrm{m}$. Each pair of neighboring genes was then scored as a positive hit if they both shared at least one voxel of the same speckle. We ran our algorithm for as many iterations $(\sim 180,000$ iterations/nucleus) as needed to obtain 1,000 neighboring gene pairs in a single nucleus ( $0.55 \%$ chance $)$. Out of these 1,000 simulated neighboring pairs, only $5.2 \pm 1.2 \%$ were positive hits (co-associating with the same speckle), which is only a $\sim 0.03 \%$ chance in total $(0.55 \times 5.2 \%)$.

Our simulation results indicate that although speckles occupy a large volume in the nucleus, merely chance co-association of genes with these large bodies can only account for $\sim 0.03 \%$ of genes in shared neighborhoods, whereas we observed a $68 \%$ rate of shared speckles for neighboring FKBP5 and SPON2. This suggests that the genes must reposition to speckles and be retained there for some amount of time in order to explain our measured association rate.

Dispersal of SC35 splicing speckles results in a significantly decreased frequency of shared neighborhoods

If SC35 splicing speckles indeed promote specific genegene positioning in induced hMADS cells, then their removal should change the spatial relation of $F K B P 5$ and $S P O N 2$. It was previously shown that overexpression of serine-arginine (SR) protein kinase cdc2-like kinase Clk 1,2 , and 3 leads to the disassembly of splicing speckles and redistribution of SC35 proteins $[45,46]$. To use the SR protein kinase as a tool to disperse the SC35 speckles in hMADS, we generated doxycycline-inducible hMDAS-CLK2GFP cells for conditional overexpression 

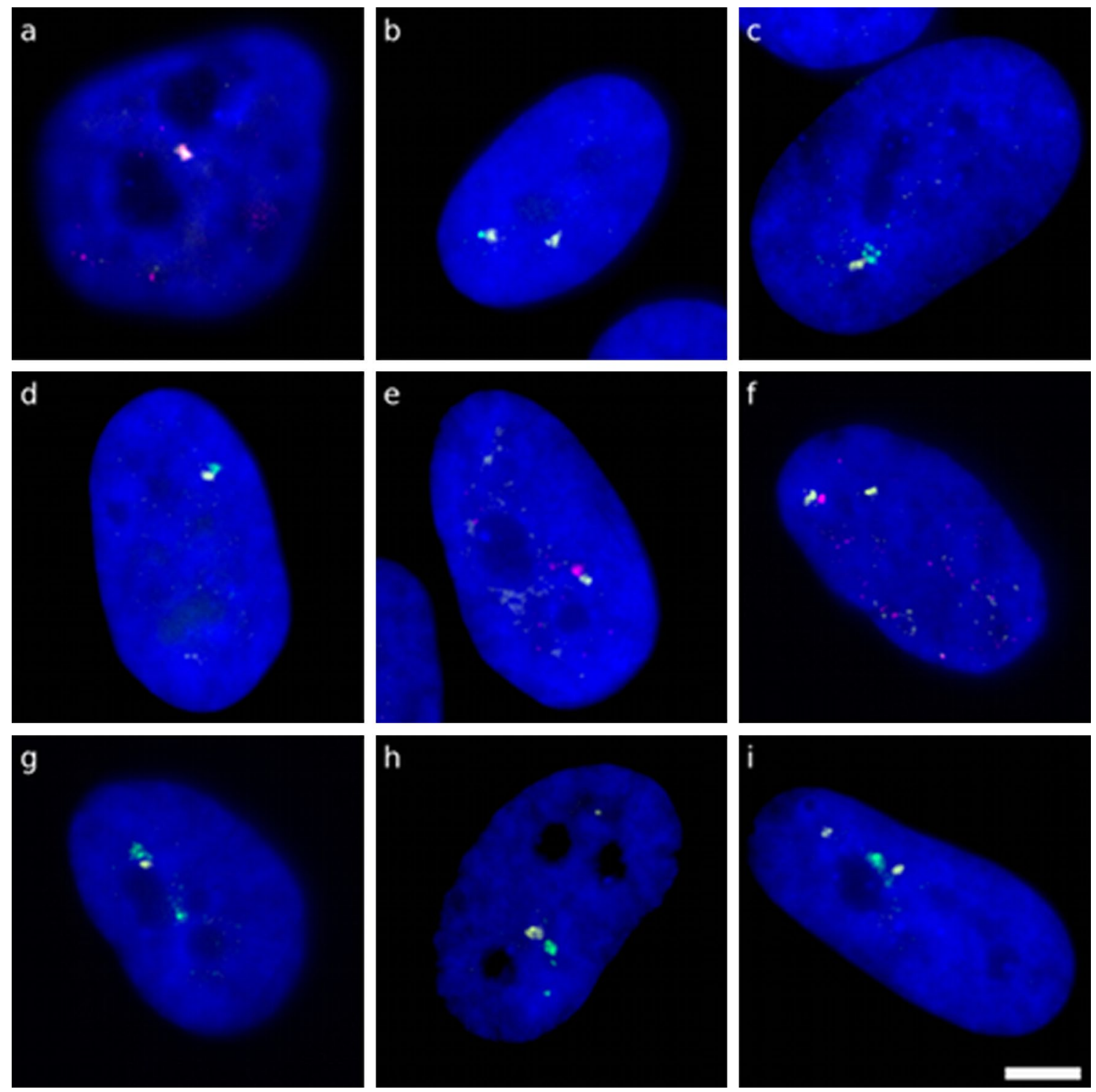

Fig. 3 RNA-FISH showing FKBP5 and SPON2 in spatial neighborhood. Representative images obtained from dual-color RNA-FISH experiments in induced hMADS cells show that transcriptionally active FKBP5 (yellow) and SPON2 (green, red in $\mathbf{a}, \mathbf{e}, \mathbf{f}$ ) are fre-

of a GFP-tagged version of human Clk2. Addition of doxycycline to these cells led to disassembly of the splicing speckles as a complete redistribution of SC35 splicing factors could be observed by immunofluorescence (supplemental information Fig. S4). We performed 3D DNA immuno-FISH in this system to study the impact of splicing speckle loss on the spatial positioning of FKBP5 and SPON2. Analogous to normal hMADS, these cells were fixed and hybridized $24 \mathrm{~h}$ after hormonal stimulation. We recorded 3D DNA immuno-FISH image stacks of both hMADS-CLK2GFP (-DOX), as shown in Fig. 5c, and CLK2 overexpressing hMADS-CLK2GFP (+DOX). From the doxycycline-treated group, we considered only quently localized in the spatial neighborhood. In such cases, the transcripts sometimes partially overlap $(\mathbf{a}, \mathbf{b})$, or show no overlap but directly contact each other $(\mathbf{c}, \mathbf{d})$, or are slightly separated $(\mathbf{e}-\mathbf{i})$ scale bar $5 \mu \mathrm{m}$

cells lacking speckles and we measured the center-tocenter distance between FKBP5 and SPON2 DNA-FISH signals in both groups $(-\mathrm{DOX} n=141,+\operatorname{DOX} n=142$, supplemental information Fig. S3f). Interestingly, we found that there was a significant (Fisher's exact test $p<0.004)$ reduction in the occurrence of close spatial positioning-dropping from $9.2 \%$ down to $1.4 \%$-when CLK2 was overexpressed and the SC35 speckles were absent (Fig. 5d), which corresponds to a $\sim 85 \%$ reduction. It should be noted that the frequency of close positioning in -DOX hMDAS-CLK2GFP cells was lower than the one we measured in normal hMADS cells, which we reason is mostly due to the higher passage number that 

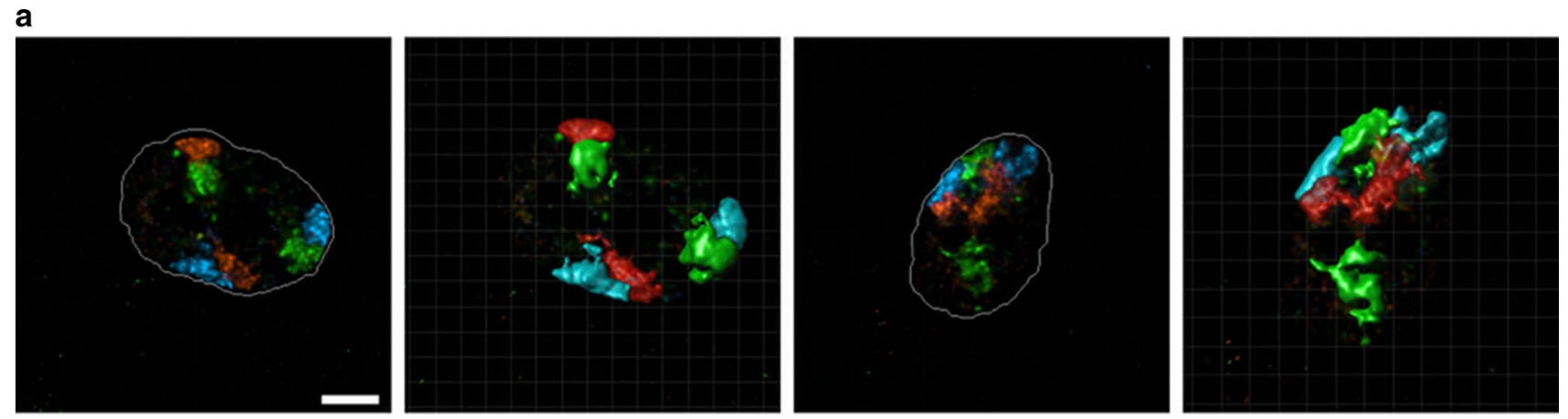

b $\quad$ CT4/CT6 distances $<4 \mu \mathrm{m}$

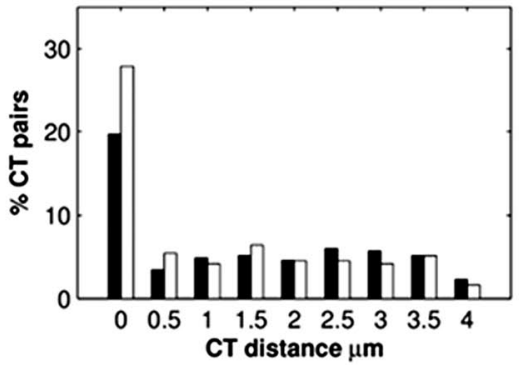

CT4/6 pair volume overlap

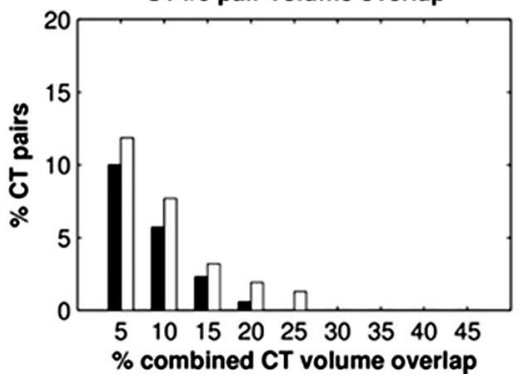

CT1/CT6 distances $<4 \mu \mathrm{m}$

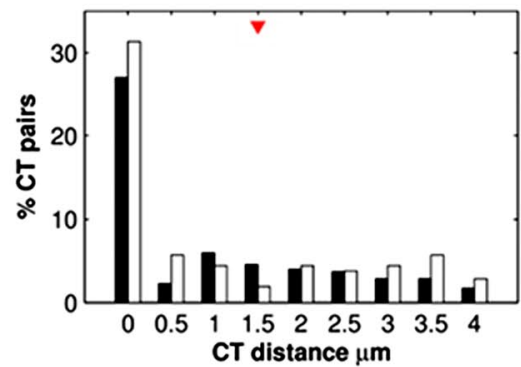

CT1/6 pair volume overlap

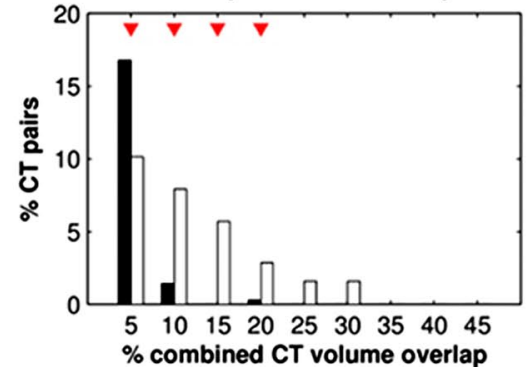

CT4/CT1 distances $<4 \mu \mathrm{m}$

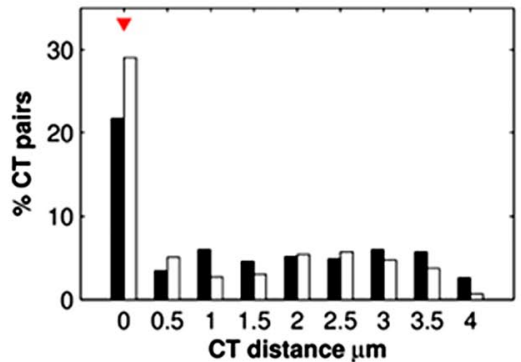

CT4/1 pair volume overlap

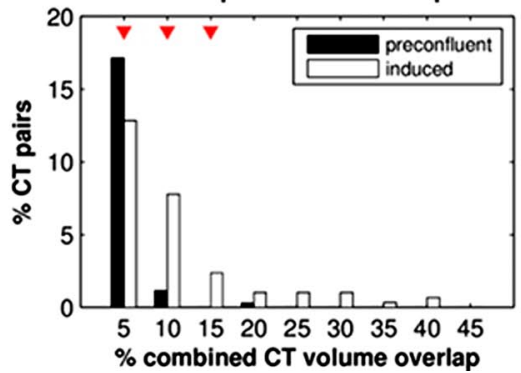

Fig. 4 Spatial organization of chromosome territories (a). Whole chromosome painting and $3 \mathrm{D}$ reconstruction of chromosome 1 (green), 4 (blue), and 6 (red) in induced hMADS cells-scale bar $=5 \mu \mathrm{m}$. The representative examples demonstrate that the chromosome territories tightly associate and intermingle. b Upper panel distribution of 3D-distances measured between each CT pair in 80 nuclei of induced (white bars) and preconfluent (black bars) hMADS cells. Shown are the percentages of chromosome territory pairs starting from either touching or overlapping (CT distance 0) up to a dis-

was reached during the generation and cultivation of the transduced cells. In earlier differentiation experiments we have seen that higher passage number hMADS cells did not respond to hormonal stimulation to the same extent as cells with low passage number.

Splicing often occurs co-transcriptionally [47], so we asked if dispersal of SC35 splicing speckles might also be affecting gene expression. We performed quantitative RTPCR with hMADS-CLK2GFP +DOX/-DOX and found that expression of the seven tested genes was decreased in the doxycycline-treated cells (supplemental information Fig. S5). Three of these genes, namely AGTR1, $F K B P 5$, and SPON2, showed a significant reduction in the tance of $4 \mu \mathrm{m}$ (binning $0.5 \mu \mathrm{m}$ ). The distributions of the individual pairs did not significantly differ from each other $(p>0.05)$. The red triangles indicate bins with statistically significant $(p<0.05)$ differences between preconfluent and induced cells. Lower panel degree of intermingling between individual chromosome territory pairs in induced (white) and preconfluent (black) cells. \% combined CT volume overlap is the co-occupied fraction of the cumulative volume of two CTs

amount of transcripts (SPON2: $-40 \%$, FKBP5: $-50 \%$, AGTR1: $-68 \%)$. We then used immuno RNA FISH (SC35/FKBP5/SPON2) to identify cells expressing FKBP5 or SPON2 and found that $<8 \%$ of the cells expressing these genes were lacking intact speckles (FKBP5: $7.9 \%$ $n=101$, SPON2: $5.8 \% n=104)$. Thus, the two tested genes are mostly expressed in cells that have intact speckles (the subpopulation of cells where co-transduction did not succeed, compare also supplemental information Fig. S4), suggesting that dispersal of speckles also affects their transcription.

In summary, these results indicate that the presence of SC35-enriched splicing speckles correlates with the 
Fig. 5 Immuno-FISH in induced hMADS cells. a An optical section where primary transcripts of FKBP5 (yellow) and SPON2 (green) are located at the same RNA polymerase II focus (purple) which was detected by the $\mathrm{H} 5$ antibody. b RNA immuno-FISH performed in induced hMADS cells shows FKBP5 (yellow) and SPON2 (green) contacting the same SC35-enriched splicing speckle (purple). c DNA immunoFISH performed in induced hMADS-CLK2GFP (-DOX) cells shows FKBP5 (red) and SPON2 (yellow) contacting the same SC35-enriched splicing speckle (blue)—scale bar $5 \mu \mathrm{m}$, the insets show zoomed details of single channels and corresponding overlays. d Frequencies at which $F K B P 5$ and SPON2 DNA-FISH signals were spatially neighboring in induced hMADS-CLK2GFP $(-\mathrm{DOX})$ before $(n=141)$, and in induced hMADS-CLK2GFP (+DOX) after complete dispersal of speckles $(n=142)$
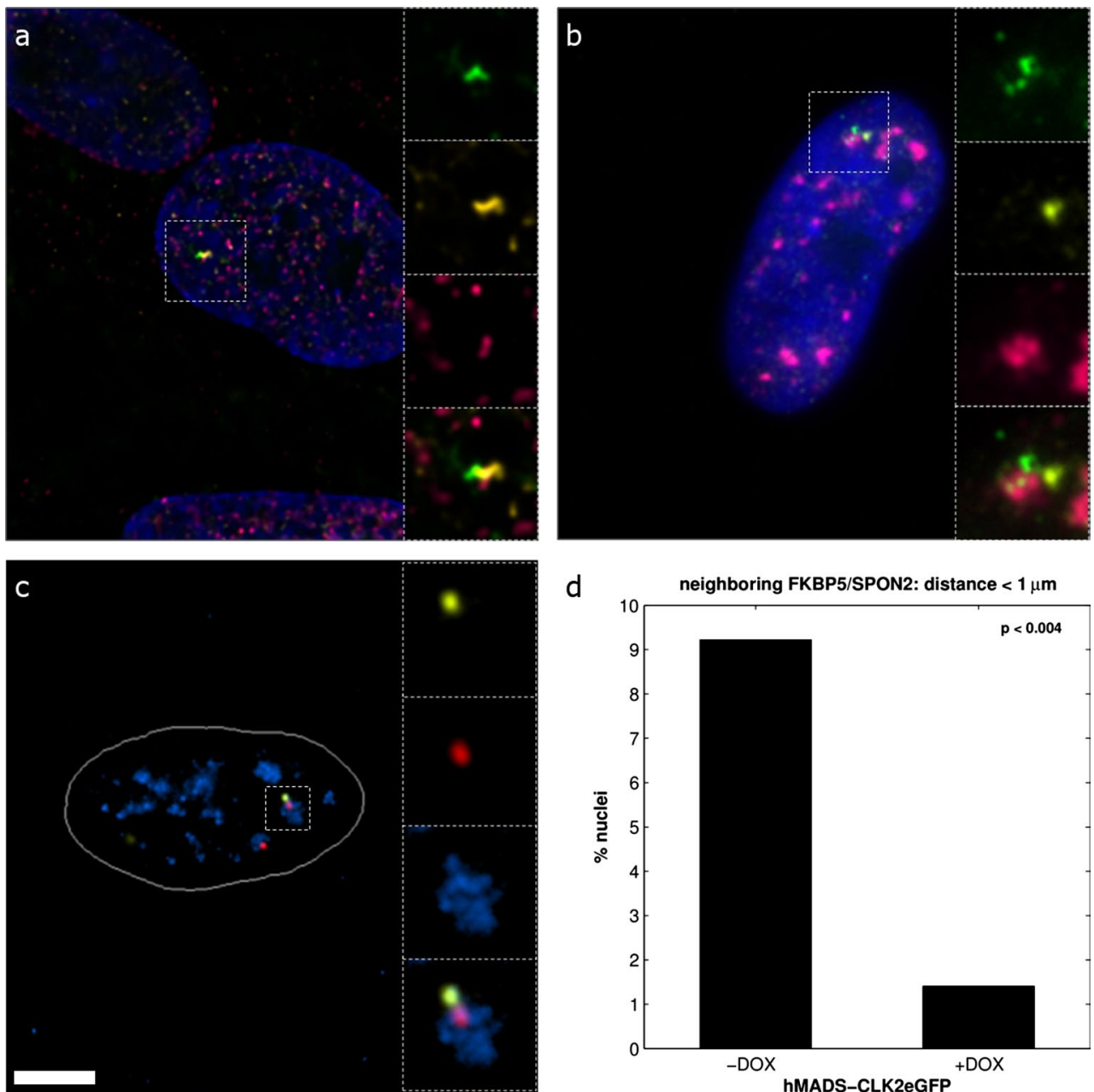

establishment of spatial gene-gene relationships in the submicrometer range and disruption of speckles decreases the transcription rate of the tested genes.

Spatially neighboring genes can share the same RNA polymerase II factories

Our analysis of speckles showed that $68 \%$ of genes in shared neighborhoods could be accounted for by association with these bodies. Their disruption led to a decreased frequency ( $\sim 85 \%$ reduction) of neighborhood sharing and negatively affected gene expression. Thus, some of this loss in neighborhood sharing could also be caused by a loss of sharing RNA polymerase II transcription factories [1, 4], which are known to mediate the clustering of active genes in erythroid cells. To determine the fraction of neighborhood sharing that is based on factory sharing of $F K B P 5 / S P O N 2$, we repeated RNA immunoFISH in induced hMADS cells using the H5 antibody against the Ser2-phosphorylated elongating form of RNA polymerase II. We found that in $17.5 \%(n=80)$ of the cells in which both genes were in shared neighborhoods, they were also associated with the same RNA polymerase II focus (Fig. 5a). From these results, we conclude that juxtapositioning of FKBP5 and SPON2, which we observed in $17.2 \%$ of the cells (Fig. 2b), can be explained by association with the same polymerase II focus in $0.175 \times 17.2=\sim 3 \%$ of the nuclei in total. Note that this number is in the same order of magnitude as found for other co-expressed genes on different chromosomes [1, 3, 4]. Thus, when we assume that also $17.5 \%$ of the $9.2 \%$ FKBP5/SPON2 neighborhood sharings in hMDAS-CLK2GFP ( - DOX) cells are due to factory sharing, we calculate a $0.175 \times 9.2=1.6 \%$ association with the same RNA polymerase II factory in total in these cells, which almost exactly corresponds to the association frequency of $1.4 \%$ that remains in hMDAS-CLK2GFP (+DOX) cells after dispersal of speckles (see Fig. 5d). This suggests that loss of factory association in such cells is not the main cause of the loss in neighborhood sharing.

In sum, we find that speckles and factories together can account for most of the active genes in shared neighborhoods, namely $\sim 68 \%$ (speckles) plus $\sim 17 \%$ (factories) or $\sim 85 \%$ of the cases of shared neighborhoods. 
Gene pre-positioning is required to account quantitatively for the observed frequencies of association with SC35 speckles and transcription factories

The simplest model for gene-gene association at a nuclear body presumes that each of the two genes must by chance be recruited to one such body in the nucleus (for example to accomplish transcription or splicing). Thus, the odds that two different genes associate with a particular body in the nucleus are inversely proportional to the number of such bodies present in that nucleus. This probability can be calculated explicitly using the formula derived by Shopland et al. [22]. To apply this formula for the case of speckle association, we first counted the average number of speckles per nucleus $(32 \pm 6)$ and also measured the frequency of speckle association individually for SPON2 (99\%) and for $F K B P 5$ (51\%). Using these measured values (see also Table 2) and the formula from Shopland et al. [22], we obtained a probability of $\sim 6.4 \% \pm 1.2 \%$ at which FKBP5 and SPON2 should be at the same speckle (Table 3). However, this is well below the observed frequency of $12 \%$, suggesting that a purely random recruitment to any one of the speckles in the nucleus cannot explain the association of these two genes.

One way to achieve a higher predicted frequency of gene-gene association at the same speckle would be to reduce the number of speckles available for association. This is a plausible possibility since we found that all seven genes were already non-randomly positioned in the nucleus before induction. Thus, if FKBP5 and SPON2 were relatively close before induction, then upon transcriptional upregulation they would be expected to sample predominantly this smaller sub-volume for an available speckle.

As a simple means to define sub-volumes for FKBP5 and $S P O N 2$, we used their radial position within the nucleus, which has been frequently used before as a measure to define preferred positioning of genes in the nucleus $[17,48,49]$. We found that $80 \%$ of the FKBP5 and SPON2 DNA FISH signals were located in the four innermost nuclear shells (Fig. 6a, b). We then assessed how often the two genes were positioned in shared neighborhoods within each shell and observed that most of the neighborhood sharing ( $91.9 \%$ which corresponds to $\sim 16 \%$ of the nuclei) also occurred in the inner four shells of the nuclear volume.

We then calculated the probability of random recruitment to the same speckle in this confined sub-volume and found that this probability increased significantly from $6.4 \pm 1.2 \%$ (whole nucleus) to $9.8 \pm 1.9 \%$ (sub-volume). This increase occurred because the average number of speckles within the sub-volume decreased to $20 \pm 4$ compared to $32 \pm 6$ in the whole nucleus (Table 2), thereby increasing the odds that the two genes would be randomly recruited to the same speckle. Now the resultant estimate of $\sim 10 \%$ for the frequency of gene associations is close to the measured frequency of $\sim 12 \%$ obtained in the preceding section by tabulating when the two loci were found at the same speckle (Table 3). Since $\sim 92 \%$ of close positioned $F K B P 5 / S P O N 2$ was located in the inner four nuclear shells, the predicted numbers are consistent with our observations $(0.92 \times 12 \%=11 \%)$. Thus, the data suggest that the close
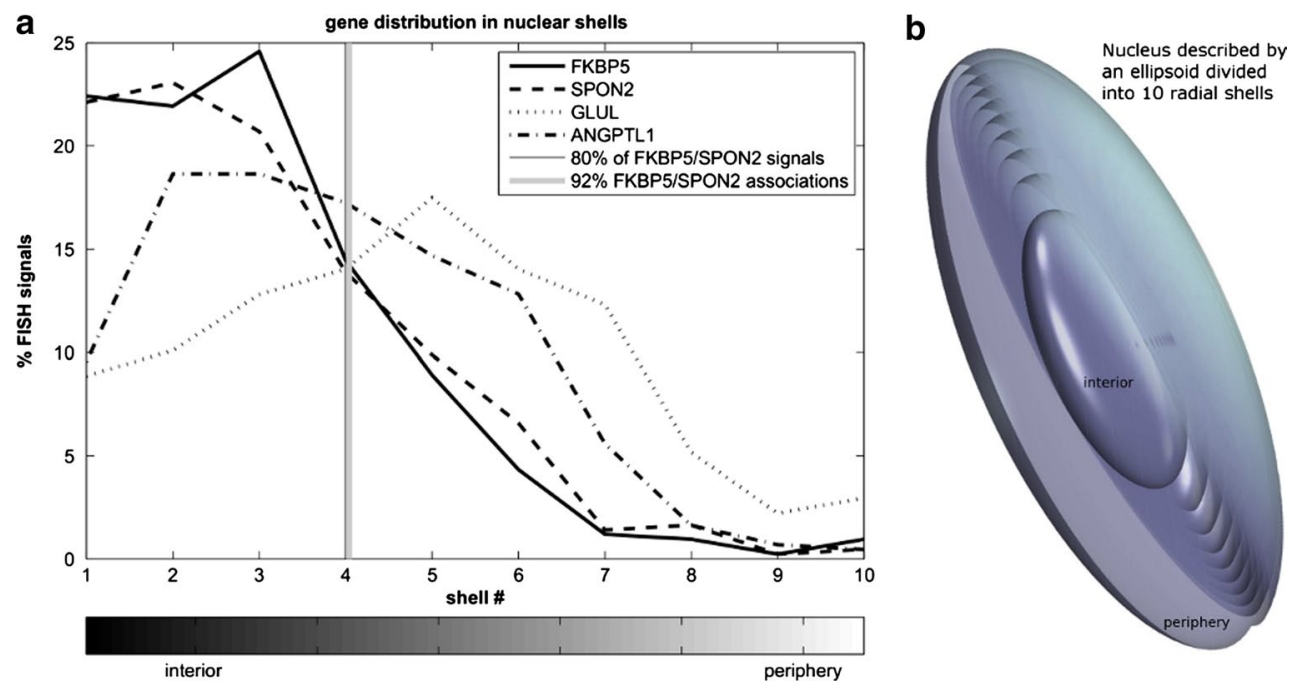

Fig. 6 Radial gene positioning. The frequency with which a gene was located in a shell was recorded and the distribution was plotted against the shell number (a). The radial position of genes was determined by assigning the corresponding DNA-FISH signals to ten radial shells of equal volume (b). FKBP5 and SPON2 were frequently found in the nuclear interior and $80 \%$ of the signals were assigned to the inner four shells where we found $92 \%$ of the spatial associations of the two genes. In contrast, the location frequency of ANGPTL1 and $G L U L$ was shifted towards to nuclear periphery and these two genes showed a very low frequency of association with either FKBP5 or SPON2 (see also Fig. $2 \mathrm{a}, \mathrm{b}$ ) 
positioning of the genes can be explained by stochastic association with nearby speckles located within a restricted nuclear sub-volume, rather than a specific or biased recruitment to a unique speckle.

We next applied the preceding model to transcription factories. As for the calculation with speckles, we measured the individual frequency of factory association for SPON2 (90.5\%) and for FKBP5 (93.5\%), plus the average number of factories in the nuclear sub-volume (Table 2) and used the values in the same formula from Shopland et al. According to this calculation, the estimated frequency of random association with a unique factory was $2.0 \pm 0.2 \%$, which is close to our measured frequency of $3 \%$ for association with the same factory (Table 3 ). The predicted lower frequency of association with the same factory compared to the same speckle is related to the increased number of factories per cell $(\sim 403 \pm 61)$ compared to the number of speckles per cell $(\sim 32 \pm 6)$. It should be noted that the $\mathrm{H} 5$ antibody that we used in immuno-FISH experiments detects most of the actively elongating transcription factories, since nearly all RNA-FISH signals ( $>90 \%$, see Table 2) were located at polymerase II foci and we counted a similar number of factories $(432 \pm 94, n=7)$ in dedicated H5 immunofluorescence stainings.

The preceding results show that we can quantitatively account for the association of two genes in a shared neighborhood based on random associations with speckles and factories within a sub-volume defined by preferred radial positioning. These preferred radial positions presumably arise because FKBP5 and SPON2 are both located in generich domains ( 89 genes within ten megabase pairs). As we have seen for FKBP5 and SPON2, such domains tend to bias genes to the nuclear interior $[49,50]$. Two other genes, GLUL and ANGPTL1, which we analyzed in our cohort, are located in gene-poor domains (59 genes within ten megabase pairs). We found that these two genes were more often positioned in the nuclear periphery (Fig. 6a). Importantly, we also found that the peripherally located genes GLUL and ANGPTL1 rarely shared the same neighborhood with the more interiorly located genes FBKB5 and SPON2 (Fig. 2a, b). These results are consistent with our model in which shared neighborhoods arise because transcriptionally active genes tend to associate with nuclear bodies in their immediate vicinity.

\section{Discussion}

\section{Overview}

We studied the spatial organization of active genes in a human stem cell model. After analyzing gene expression profiles during the transition from uncommitted cells to mature adipocytes, we identified co-expressed genes and then asked if they showed spatial organization within the nucleus. We examined seven co-expressed genes and found that they were pre-positioned relative to each other according to a non-random topology. This organization was preserved between different nuclei and did not change dramatically after hormonal stimulation.

Within this global organization, we then investigated pair-wise relationships between the co-expressed genes. We found that upon hormonal stimulation and concomitant transcriptional upregulation, five of the seven genes shared 1- $\mu \mathrm{m}$-diameter neighborhoods with another co-transcribed gene at frequencies higher than expected by chance. Except for GLUL and ANGPTL1, the studied genes were not near each other on the same chromosomes, so these shared gene-gene neighborhoods must have arisen from some set of organizational principles.

Such gene-gene associations have been studied before and different models have been proposed to explain them (e.g., $[1-4,15])$. Our results suggest a new hybrid model that combines features from several previously proposed and apparently contradictory models. We find that prior to induction the genes are prepositioned such that upon transcriptional upregulation they are more likely to encounter nearby speckles and transcription factories. This initial prepositioning thereby increases the odds of sharing the same SC35 splicing speckle or RNA polymerase II transcription factory to a level that agrees quantitatively with the frequency of shared neighborhoods that we have measured.

Factors influencing shared gene-gene neighborhoods

We defined a shared neighborhood as two genes that were within a distance of $<1 \mu \mathrm{m}$. This is approximately the diameter of an average nuclear body so it allowed us to investigate how association with nuclear bodies can influence gene positioning within the nucleus. Note however that genes positioned within $1 \mu \mathrm{m}$ of each other in a fixed cell will occasionally be much closer in live cells due to the diffusion of chromatin, which is known to occur in the submicron to micron range [51]. Indeed, it has been shown that interaction signals measured by $4 \mathrm{C}$ robustly correlated with distances of up to $1 \mu \mathrm{m}$ as measured by 3D DNA-FISH [52].

We first considered the naive explanation that intermingling of CTs $[42,44]$ could explain the frequencies at which gene pairs were found in the same spatial neighborhood. However, we found that the spatial relations between CTs bearing a pair of genes (FKBP5/SPON2) that frequently shared the same neighborhood did not differ significantly from the spatial relation between CTs carrying genes that did not share the same neighborhood. This suggests that CT positioning alone is not sufficient to explain 
the observed differences in neighborhood sharing and indicates that additional factors might specifically situate genes in shared neighborhoods.

The frequency at which genes were located in shared neighborhoods rose upon increased expression levels and decreased upon inhibition of transcription, hence we tested if genes in a spatial neighborhood were associated with nuclear bodies involved in transcription. We focused on two different nuclear bodies that were previously considered to mediate spatial gene organization, namely SC35 splicing speckles $[2,3]$ and RNA polymerase II transcription factories $[1,4,15]$.

We found that in $68 \%$ of the cases in which FKBP5 and SPON2 were in a spatial shared neighborhood they also associated with the same splicing speckle. It is important to note that these bodies are large in size $(\sim 0.5$ to $\sim 2 \mu \mathrm{m})$. We therefore considered the possibility that pure-chance co-association of genes with a large volume of speckles is enough to explain the measured association frequencies. A computer simulation of this model, however, could not reproduce observations we made in hMADS cells. Thus, it seems unlikely that the two genes share the same neighborhood only by chance; rather it suggests that it is mediated by the presence of speckles.

In fact, we showed that the loss of speckles significantly reduces the frequency at which FKBP5 and SPON2 are found in shared neighborhoods, suggesting that the presence of speckles promotes spatial gene-gene association. It is known that most splicing is occurring co-transcriptionally [47] and that the splicing factor SC35 has an important role in transcriptional elongation [53]. Association of highly active genes with the periphery of speckles brings them to an environment with highly increased concentrations of splicing factors and therefore enables more efficient transcription and processing. Accordingly, in CLK2 overexpressing cells, we measured decreased expression of the seven tested genes. Moreover, FKBP5 and SPON2 were almost exclusively expressed in cells that had intact speckles (= subpopulation of cells where co-transduction did not succeed, see Fig. S3), while $<8 \%$ of cells expressing theses genes were lacking intact speckles. In addition to the reduction in the local concentration of splicing factors, the constant overexpression of CLK2 (48 $\mathrm{h}$ in our experiments) may lead to imbalanced phosphorylation states of SR proteins with negative effects on splicing processes and in further consequence also on transcription.

Besides speckles, which could account for mediating a substantial fraction of shared neighborhoods, we found a second fraction $(17.5 \%)$ of nuclear neighborhoods in which FKBP5 and SPON2 shared the same transcription factory. In fixed cells, factory sharing accounted for the most closely positioned pairs, separated by no more than the diameter of a factory $(40-198 \mathrm{~nm}[54,55])$. It is possible that our measurement of this $17.5 \%$ fraction ( $3 \%$ of all inspected cells) might be an underestimate, since a recent study found that elongating RNA polymerase II phosphorylated at Ser2 may move out of factories following initiation or early elongation [56]. In addition, it was suggested that two types of transcription factories exist: "poised factories", containing RNA polymerase II phosphorylated on Ser5, but not Ser2, and "active factories", which are phosphorylated on both residues [57]. Here we used the H5 antibody, which is directed against the Ser2 phosphorylated form of RNA polymerase II and so we exclusively detected the active type of factories. Moreover, it was previously shown that genes may be localized at polymerase II foci even in the absence of transcriptional elongation [58], suggesting association with poised factories. In line with this observation, it was also reported that the DNA:DNA interactions of different loci as measured by $3 \mathrm{C}$ and $4 \mathrm{C}$ were still present after DRB treatment [19]. When we treated induced hMADS cells with DRB, we observed a significant drop in the frequency at which $F K B P$ and SPON2 shared the same $1 \mu \mathrm{m}$ neighborhood, however, there was still a fraction $(\sim 2.7 \%)$ that was closely associated (center to center distance $<0.5 \mu \mathrm{m}$ ). It is possible that this fraction of $F K B P 5 / S P O N 2$ pairs in our DRBtreated hMADS cells corresponds to the genes that had been sharing the same active- but now poised-transcription factory.

In sum, we found that both speckles and factories could contribute to co-association of the FKBP5 and SPON2 genes in shared neighborhoods. Note that because these genes are sometimes transcribed at the same speckle and other times shared the same factory, their coordinate expression is not likely caused by their association with a particular body. Rather, it seems more likely that their association with these bodies is caused by their coordinate expression since genes that are transcriptionally active at the same time are more likely to be randomly recruited to the same body. Hence, it seems unlikely that sharing the same nuclear body is essential for their coordinate expression or is of significant advantage for the cell.

Quantitative analysis of gene-gene co-association supports a hybrid model

To quantitatively predict the gene-gene co-association at a specific body (speckle or factory), we first presumed a model in which two genes are recruited to any of the bodies present in a nucleus. The probability for sharing a body was calculated based on a formula derived from Shopland et al. [22]. We found that this simple model could not account for the observed frequencies at which two neighboring genes shared a nuclear body. Our analysis on the global gene topology though, suggested a non-random positioning, 
which does not change dramatically upon hormonal stimulation. In addition, we observed gene density related to radial positioning $[49,50]$, e.g., FKBP5 and SPON2, which are both located in gene-rich domains, were often positioned in the nuclear interior, whereas GLUL and ANGPTL1, which are located in gene-poor domains showed peripheral nuclear positioning (Fig. 6a). When we considered the information about radial positioning in our calculation, the number of bodies in the radial reach of a gene was reduced. Consequently the probability that two neighboring genes are recruited to a shared body increased, which resulted in a fairly good prediction of the measured values (Table 3 ). Specifically, we estimate that the model can account for $\sim 75 \%$ of the observations in which FKBP5 and SPON2 were positioned in spatial neighborhoods. This estimate was obtained by accounting for both speckle and factory sharing, minus the overlap between these two populations (see supplemental information for details of this calculation).

Taken together, we propose that a non-random spatial organization prepositions genes into preferred nuclear subvolumes and association with factories or SC35 speckles occurs stochastically within the radial range of a gene. In this model, the frequency of co-association with a shared body is determined by the limiting number of bodies within the nuclear sub-volume.

\section{Application of the proposed model}

Our proposed model for shared gene neighborhoods may help account for the disparate results reported in several previous studies where either speckles [2,3] or transcription factories $[1,4,15]$ were identified as the predominant body mediating specific gene positioning. The Osborne and Schoenfelder studies examined mouse erythroid cells where there are relatively few polymerase factories $(\sim 100$ $300)$ and no well-defined speckles. Their observation that gene-gene associations occur at shared factories could be expected, given the limited number of factories. Further restricting the number of factories is the fact that "specialized" transcription factories-containing a specific transcription factor-are preferentially shared, as Schoenfelder et al. have suggested. Conversely, in the erythroid cells examined by Brown et al., the fibroblast cells examined by Shopland et al., and the porcine adipocytes examined by Szczerbal and Bridger, well-defined speckles exist in relatively small numbers $(\sim 15-40)$. Although Shopland et al. [22] as well as Szczerbal and Bridger [23] did not report the number of factories in their cells, Brown et al. [3] estimated a large number $(\sim 1,100-1,900)$. Thus, at least in the erythroid cells examined by Brown et al., co-associations with speckles would be expected to predominate since there are far fewer speckles than factories.
Conclusions and outlook

In conclusion, we provide a straightforward explanation for our general observations of spatial organization of active genes in human stem cells with a model that may also be able to account for observations in other eukaryotic cells. Thus, it will be interesting to apply the procedures we have developed here to a larger set of genes in different co-regulatory clusters as well as to genes in other cell systems, in order to test whether the model can capture the basic principles of spatial organization of active genes in a wide variety of genes and cell types.

It is important to note that the approach of 3D FISH that we have used here allows for the analysis of genegene associations within single cells. This is in contrast to a number of recent genome-wide studies using 3C-based high-throughput methods like $\mathrm{HiC}, 4 \mathrm{C}$, e4C, and ChIAPET, which have revealed a large cohort of potential chromatin interactions and spatial topologies [4, 18-21, 43, 52, 59]. Many of these interactions were cell type-specific and are thought to present the basis for cell-specific regulation of transcription. While these are powerful approaches, it will still be important in the future to continue to examine relationships within individual cells. Here high-throughput imaging of 3D FISH preparations could reveal the diversity across the cell population. Combined with the quantitative analyses that we have developed here, this should allow for a more thorough dissection of the rules governing nuclear organization.

\section{Materials and methods}

Gene selection by microarray data analysis

We examined microarray expression profiles [34] to select seven genes. Briefly: co-expressed genes were determined by the $\mathrm{k}$-means clustering $(k=12)$. Cluster 10 (supplemental information Fig. S1) was further subdivided $(k=7)$ to obtain seven genes with different genomic locations: C4orf18-Chr $4159.3 \mathrm{Mbps}$, GLUL-Chr 1 $180.6 \mathrm{Mbps}$, SAMHDI-Chr $2035.0 \mathrm{Mbps}$, AGTRI-Chr 3149.9 Mbps, ANGPTL1-Chr 1177.1 Mbps, FKBP5Chr 635.7 Mbps, SPON2—Chr 4 1.1 Mbps.

\section{Cell culture}

hMADS cells were grown on coverslips as described in [34]. Preconfluent cells were fixed at 80-90\% confluence. For induction, the medium was exchanged as previously described [34] and the cells were fixed after 24 h. For Clk2 overexpression, hMADS-CLK2GFP were treated likewise 
and $\sim 24 \mathrm{~h}$ before induction of adipogenesis $1 \mu \mathrm{g} / \mathrm{ml}$ doxycycline was added to the medium.

Generation of hMDAS-CLK2GFP cells for conditional overexpression of $C L K 2$

The lentiviral plasmid for conditional overexpression of human CLK2 was generated by LR-recombination (GATEWAY, Life Technologies, Carlsbad, CA/USA) of pDONR223-CLK2 (Addgene, Cambridge, MA/USA) with pHR-TetCMV-DEST-IRES-mGFP5 as previously described [60]. For the production of lentiviral particles, HEK293T cells were transfected either with $5 \mu \mathrm{g}$ pHR-TetCMVhCLK2-IRES-mGFP5, $5 \mu \mathrm{g}$ pHR-SFFV-TetRKRAB-IRESPuro [60], or pHR-SFFV-rtTAM2-T2A-Puro (Ploner et al., in prep) together with $3 \mu \mathrm{g}$ pSPAX2 and $3 \mu \mathrm{g}$ pMD-G (kindly provided by D. Trono). hMADS cells were first transduced with lentiviral particles encoding TetR-KRAB and rtTAM2 sequences to enable tetracycline (doxycycline) controlled expression of hCLK2 in a tight system (co-transduction with tetR-KRAB). Puromycin-resistant hMADS cells were further superinfected with lentiviral particles encoding hCLK2cDNA under the control of the doxycycline-sensitive TetCMV promoter (pHR-TetCMV-hCLK2-IRES-mGFP5).

\section{Cell fixation and permeabilization}

The nuclei preservation procedure used in our study is based on fixation with PBS buffered paraformaldehyde (PFA), which was shown to sufficiently preserve largescale chromatin structures at the level of conventional [61, 62] and super-resolution [63] light microscopy. We avoided the frequently used hypotonic methanol/acetic fixation since it is known that this method strongly affects the nuclear and chromatin morphology, instead we used a long (25 min) fixation in $3.5 \%$ PFA following a short (1 min) permeabilization in CSK buffer $(100 \mathrm{mM} \mathrm{NaCl}, 200 \mathrm{mM}$ sucrose, $10 \mathrm{mM}$ PIPES $\mathrm{pH} 6.8,3 \mathrm{mM} \mathrm{MgCl}_{2}$ ) on ice supplemented with $0.5 \%$ Triton $\mathrm{X}-100$. The cells were then washed $2 \times 5$ min in PBS.

3D combinatorial M-FISH

\section{Probe generation}

BAC-DNA(ImaGenes GmbH, Berlin, Germany: RP11-89C4 for C4orf18, RP1-223H12 for GLUL, RP1-132F21 SAMHD1, RP11-505J9 for AGTR1, RP4-595C2 for ANGPTL1, RP3-368C2 for FKBP5 and RP11-20I20 for $S P O N 2)$ was isolated and labeled by nick translation with BIO-16-dUTP (biotin), DIG-11-dUTP, (digoxigenin) or DNP-11-dUTP (dinitrophenyl). We used the three labels in all possible combinations ( $n=3,2^{n}-1=7$ genes).

\section{Hybridization mix}

The hybridization mix was composed of $100 \mathrm{ng}$ of each nick-translated probe, $56 \mu \mathrm{g}$ salmon sperm DNA, and $40 \mu \mathrm{g}$ human cot1 DNA (Life Technologies, Carlsbad, CA/USA) in $50 \%$ formamide $/ 2 \times$ SSC $5 \%$ dextran sulfate. Prior to hybridization, the DNA was kept for $10 \mathrm{~min}$ at $85^{\circ} \mathrm{C}$ and pre-hybridized for $20 \mathrm{~min}$ at $37^{\circ} \mathrm{C}$.

\section{In situ hybridization}

In situ hybridization of was carried out as described in [64] but with the following modifications: cells were treated with RNase for $20 \mathrm{~min}$, chromatin was denaturated for 4.5 min at $85{ }^{\circ} \mathrm{C}$ in $70 \%$ formamide $/ 2 \times$ SSC directly followed by the ethanol series and hybridized over two nights at $37^{\circ} \mathrm{C}$. After the hybridization, a series of washes was performed: $2 \times 15 \mathrm{~min} 50 \%$ formamide $2 \times \mathrm{SSC}$ at $45{ }^{\circ} \mathrm{C}$, $10 \min 0.1 \times \mathrm{SSC}, 10 \min 2 \times \mathrm{SSC}$, and $5 \min 4 \times \mathrm{SSC}$.

\section{Detection of the probes}

Biotin labels were detected with streptavidin Alexa Fluor 647 (Life Technologies, Carlsbad, CA/USA) diluted 1:200 in $4 \times \mathrm{SSC} / 0.1 \% \mathrm{BSA} / 0.01 \%$ Tween 20 . Washes in $4 \times \mathrm{SSC} / 0.1 \%$ Tween 20 for $10 \mathrm{~min}, 4 \times \mathrm{SSC}$ for $2 \times 10 \mathrm{~min}, 2 \times \mathrm{SSC}$ for $10 \mathrm{~min}$, and PBS for $5 \mathrm{~min}$ followed the incubation step. The DIG and DNP labels with anti-digoxigenin-rhodamine (Roche Applied Science, Penzberg, Germany) and anti-dinitrophenyl-KLH Alexa Fluor 488 antibodies, diluted 1:200 in $4 \%$ BSA/0.1 \% Tween 20/ PBS. After washing $3 \times 5$ min with PBS, the nuclei were counterstained with $0.05 \mu \mathrm{g} / \mathrm{ml}$ DAPI in PBS for $5 \mathrm{~min}$.

\section{RNA-FISH}

The intron probes for FKBP5 and SPON2 were generated by PCR using specific primers (supplemental information Table 1). The PCR products were amine-modified by nicktranslation using amino-allyl-dUTP, which was then coupled to an amine-reactive fluorescent dye (Cy3 for FKBP5, Alexa Fluor 488 for SPON2) following the ARES DNA Labeling Kit (Life Technologies, Carlsbad, CA/USA) instructions.

\section{In situ hybridization}

Fixed and permeabilized nuclei were dehydrated by a series of washes in 70, $90 \%$, and absolute $\mathrm{EtOH}$ on ice for $5 \mathrm{~min}$ each. The cells were air-dried and hybridized overnight at $37{ }^{\circ} \mathrm{C}$ with $5 \mu$ l of the hybridization-cocktail (FKBP5, SPON2 $20 \mathrm{ng}$ each, $2 \times \mathrm{SSC}, 5 \%$ dextran sulfate, $1 \mathrm{mg} / \mathrm{ml}$ yeast t-RNA, $50 \%$ formamide). After hybridization, the coverslip was washed $3 \times 5 \mathrm{~min}$ in $50 \%$ 
formamide $/ 2 \times \mathrm{SSC}$ at $37{ }^{\circ} \mathrm{C}, 15 \mathrm{~min} 2 \times \mathrm{SSC}$, and $5 \mathrm{~min}$ in PBS. The nuclei were counterstained with $0.05 \mu \mathrm{g} / \mathrm{ml}$ DAPI in PBS for 5 min.

\section{RNA immuno-FISH}

The mouse H5 antibody (Convance, Princeton, NJ/USA; Abcam, Cambridge, UK) and a mouse anti-SC35 (Abcam, Cambridge, UK) was used to detect active RNA polymerase II and splicing speckles. The antibodies were diluted 1:200 in $4 \%$ BSA/0.1\% Tween 20/PBS containing $2 \mu 1$ RNAse OUT (Life Technologies, Carlsbad, CA/USA) and incubated overnight at $4{ }^{\circ} \mathrm{C}$. After three washes with PBS, an anti-mouse Cy5 secondary antibody (Abcam, Cambridge, UK) was diluted as described above, applied for $1 \mathrm{~h}$ at room temperature, and washed three times. The signal was fixed for 10 min with $3.5 \%$ PFA in PBS. The RNA hybridization was performed as described above but the dehydration steps were omitted.

\section{DNA immuno-FISH (SC35/FKBP5/SPON2)}

\section{Probe generation}

BAC-DNA (ImaGenes GmbH, Berlin, Germany: RP3$368 \mathrm{C} 2$ for $F K B P 5$ and RP11-20I20 for SPON2) was isolated and sonicated to a size of $200-500$ base pairs using the Covaris S2 sonicator. The 500 ng of DNA fragments were directly labeled with AlexaFlour 3 (SPON2) and AlexaFluor 5 (FKBP5) using the BioPrime labeling system (Life Technologies, Carlsbad, CA/USA).

\section{In situ hybridization and immunostaining}

Cells grown on coverslips were fixed and permeabilized as described above followed by 5-min incubation with $0.1 \mathrm{~N}$ $\mathrm{HCl}$. After washing with PBS, the cells were treated for 30 min with RNAse $(20 \mu \mathrm{g} / \mathrm{ml})$, washed again, and incubated in $2 \times \mathrm{SSC}$ for $10 \mathrm{~min}$ and then in $2 \times \mathrm{SSC} / 70 \%$ deionized formamide for $45 \mathrm{~min}$. Then the coverslips were directly applied upside down onto the hybridization mix (120 ng probe for both, FKBP5 and SPON2, $6 \mu \mathrm{g}$ human cot1 DNA, $20 \mu \mathrm{g}$ salmon sperm DNA in $50 \%$ formamide $/ 2 \times \operatorname{SSC} 5 \%$ dextran sulfate). The coverslip was sealed with rubber cement before denaturating for $4.5 \mathrm{~min}$ at $85{ }^{\circ} \mathrm{C}$. The cells were hybridized at $37{ }^{\circ} \mathrm{C}$ overnight and washed $1 \times 15 \operatorname{min~} 50 \%$ formamide $/ 2 \times \operatorname{SSC} 42{ }^{\circ} \mathrm{C}$, $10 \min 0.1 \times \mathrm{SSC}, 10 \mathrm{~min} 2 \times \mathrm{SSC}$ and $5 \mathrm{~min}$ in PBS. SC35 splicing speckles were detected as described in the RNA immuno-FISH section but with the following modifications, addition of RNAse OUT was omitted and an anti-mouse AlexaFluor 488 secondary antibody was used. Primary as well as secondary antibodies were incubated for
$1 \mathrm{~h}$ at $37^{\circ} \mathrm{C}$ or overnight at $4{ }^{\circ} \mathrm{C}$. The nuclei were counterstained with $0.025 \mu \mathrm{g} / \mathrm{ml}$ DAPI in PBS for $5 \mathrm{~min}$.

Chromosome painting

DOP-PCR amplified flow-sorted painting probes [65] for chromosomes 1, 4, and 6 (Applied Spectral Imaging Carls$\mathrm{bad}, \mathrm{CA} / \mathrm{USA}$ ) were hybridized following the procedure described in [66] with modifications: fixation: $15 \mathrm{~min}$ in $3.5 \%$ PFA, 15-min permeabilization, over-night equilibration in $2 \times \mathrm{SSC} / 70 \%$ deionized formamide, chromatin denaturation $3 \mathrm{~min}$ at $72{ }^{\circ} \mathrm{C}$. After an ethanol dehydration series, the coverslips were air-dried for $5 \mathrm{~min}$ and $2 \mu \mathrm{l}$ of each painting probe-which were heat denaturated for $10 \mathrm{~min}$ at $80^{\circ} \mathrm{C}$ and pre-hybridized for $20 \mathrm{~min}$ at $37^{\circ} \mathrm{C}-$ was applied, and hybridized over two nights at $37^{\circ} \mathrm{C}$. Two stringency washes were performed: $4 \mathrm{~min}$ in $0.4 \times \mathrm{SSC}$ at $74{ }^{\circ} \mathrm{C}, 2 \mathrm{~min} 4 \times \mathrm{SSC} / 0.1 \%$ Tween 20 .

Immunofluorescence

SC35 splicing speckles: Cells were fixed in $3.5 \%$ PFA/ PBS for $10 \mathrm{~min}$ and permeabilized for $15 \mathrm{~min}$ with $0.5 \%$ Triton X-100 in PBS. Immunostaining of SC35 was performed as described in the DNA immuno-FISH section, but with the following modifications, an anti-mouse AlexaFluor 546 secondary antibody was used. RNA polymerase II (Ser2 phosphorylated): Cells were fixed for $20 \mathrm{~min}$ in $3.5 \%$ PFA/PBS following a 1-min permeabilization in CSK buffer. Immunostaining was performed as described in the RNA immuno-FISH section.

\section{BrUTP incorporation}

The protocol followed that in [67], with the following modifications. The permeabilization buffer contained $10 \mu \mathrm{g} / \mathrm{ml}$ instead of $25 \mu \mathrm{g} / \mathrm{ml}$ digitonin, dexamethasone was omitted. The transcription reaction was run for $15 \mathrm{~min}$ at $37^{\circ} \mathrm{C}$. RNaseOUT (Invitrogen) was used instead of RNasin. Cells were fixed in $2 \%$ PFA/PBS for $10 \mathrm{~min}$ after a 30-s permeabilization in CSK buffer $+0.5 \%$ Triton X-100. Incorporated BrUTP was detected using the mouse anti-BrdU (sc-32323, Santa Cruz Biotechnology, Inc., Santa Cruz, CA/USA) and an anti-mouse AlexaFluor 488 secondary antibody.

Transcription inhibition experiments

Transcriptional elongation was inhibited by incubation in growth medium supplemented with $50 \mu \mathrm{g} / \mathrm{ml}$ DRB (Sigma-Aldrich, St. Louis, MO/USA). After 1-h incubation, the cells were prepared for DNA-FISH or BrUTP incorporation. Inhibited cells could be rescued by $45 \mathrm{~min}$. Incubation in normal growth medium. 
Quantitative real-time reverse transcriptase PCR (qPCR)

For quantitation of mRNA transcripts in hMADS cultures, the qRT-PCR standard curve method was applied. Standards were prepared by amplicon-selective PCR performed on total cDNA generated from hMADS total RNA. Obtained amplicons were purified and quantitated with Nanodrop (Peqlab, Erlangen, Germany). Standard concentrations in the range 100-0.0001 pM amplicon were applied for qRT-PCR together with the samples to be assayed, applying Platinum SYBR Green, the Viia7 realtime PCR technology and software from Life Technologies (Carlsbad, CA/USA). Primers for generating the standards and for the real-time PCR are found in supplemental information Table 1.

Microscopy, image, and data analysis

\section{Microscopic image acquisition}

Images were recorded on a Zeiss AxioImager Z1 epifluorescence microscope equipped with an AxioCam MRm CCD camera and a Heidenhain closed-loop system for highly precise and reproducible z-stacks of equidistant step sizes. The 3D-image stacks were collected using a $100 \times$ Plan-Apochromat 1.4 NA oil immersion lens with an axial sampling distance of $200 \mathrm{~nm}$ producing a voxel size of $64.3 \times 64.3 \times 200 \mathrm{~nm}$. In case of DNA mFISH, nuclei were chosen based on the DAPI stain and the presence of 8 Alexa Flour 488 signals. This minimized a potential bias since the combinatorial labeling of the seven genes was not revealed by looking at only one fluorochrome. In case of RNA-FISH and RNA immuno-FISH, nuclei were chosen by the presence of two hybridization signals.

\section{Image restoration and $3 D$ reconstruction}

All recorded image stacks were processed by 3D-deconvolution running 100 iterations of the maximum likelihood estimation algorithm available with the Huygens Deconvolution Software (Scientific Volume Imaging -SVI http://w ww.svi.nl). The deconvolved image stacks were then corrected for color-shift errors.

For the detection and measurement of the FISH-signals, the processed image stacks were reconstructed in 3D. The reconstruction method was realized with Imaris (Bitplane Inc. South Windsor, CT/USA) using the ImarisXT interface and the Matlab (MathWorks Inc. Natick, MA/USA) programming language, which also included the image processing and statistics toolboxes. The procedure involved the following five steps:

(1) Removal of nuclei touching the lateral image borders, (2) generation of an isosurface in the DAPI channel that delineates the nucleus, (3) removing any extra-nuclear background by setting voxel intensities outside of the nuclear surface to zero in all channels, (4) detection of FISH signals by using the "DetectSpot" function of ImarisXT. By dynamically adjusting the intensity threshold, the expected number of Spots (eight per channel) could be detected, (5) identification of the combinatorial labeled genes by resolving the combinatorial labeling. This was achieved by $k$-means clustering of all detected spot coordinates from each channel. The $k$-value was thereby set to the number of genes detected by our approach, usually 14 (7 genes $\times 2$ alleles). The corresponding gene names were then assigned to the centroids of the resulting cluster.

The reconstructed 3D image was used to generate the following data: the 3D coordinates of the detected genes, the center-to-center distances between the single genes, the volumes of the nuclei, and the axes' lengths including the rotation matrix of the ellipsoid that approximates and describes a nucleus' surface.

\section{Radial positioning}

Each nucleus was approximated by an ellipsoid, which was subdivided into ten shells of equal volume and shape. The frequency of gene localization in each shell was determined over all nuclei.

\section{Frequency of random gene co-association with an SC35 speckle or transcription factory}

We calculated the expected frequency of random association for one copy of a gene "a" with an SC35 speckle marked by another gene "b" using the following formula as described in [22]: ef $=\left(\frac{1}{\mathrm{nS}}\right) \times f_{\mathrm{a}} \times f_{\mathrm{b}} \times 4$, where $\mathrm{nS}=$ the average number of speckles per cell and $f_{\mathrm{a}}, f_{\mathrm{b}}=$ the independent frequencies of domain association for each gene. Based on the rules for propagation of error [68], we calculated the standard deviation for ef as $\frac{f_{\mathrm{a}} \times f_{\mathrm{b}} \times 4}{\mathrm{nS}^{2}} \times \sigma(\mathrm{nS})$, where $\sigma(\mathrm{nS})$ was the measured standard deviation in the number of speckles per cell. The same formulae were used to determine the expected co-association frequencies with RNA polymerase II transcription factories. The average number of speckles or factories was determined by automatic image segmentation, and for the restricted nuclear domain calculations only the number of bodies in the inner four shells was counted.

\section{Correlation of nuclear gene organization}

We measured the center-to-center distance between FISH signals of each of the seven genes and used the shortest distance between all alleles of a given pair $(2+2$ alleles $)$ for the analysis. For the general comparison of spatial gene-gene relations, the distances were normalized for the 
volume of the nucleus in which they were measured [69]. To determine whether the spatial organization of different nuclei correlates, we calculated the pairwise Pearson correlation coefficients of vectors containing the volumenormalized gene-gene distances. Only nuclei with no missing values were included in this analysis. Correlations were considered as significant if the $p$ value of the correlation coefficient was smaller than 0.05 . To test for the possibility that the observed correlations between nuclei could be obtained by a random assignment of the measured gene-gene distances, we randomly permuted the values of the inter-gene distance within the vectors representing a nucleus and recalculated the correlation coefficients. In addition, we repeated this analysis using random genegene distances $(0 \leq d \leq$ mean diameter of hMADS nuclei).

Acknowledgments This work was supported by the Austrian Federal Ministry for Science and Research, Project GEN-AU BIN and the Austrian Science Funds, Projects SFB Lipotoxicity and SFB Cell proliferation and cell death in tumors. We thank Martina Wick, Claudia Neuhold, and Nicole Golob for support in the lab, and Hubert Hackl for statistical advice and valuable comments.

Conflict of interest The authors declare no conflicts of interest.

\section{References}

1. Osborne CS, Chakalova L, Brown KE et al (2004) Active genes dynamically colocalize to shared sites of ongoing transcription. Nat Genet 36:1065-1071

2. Brown JM, Leach J, Reittie JE, Atzberger A, Lee-Prudhoe J, Wood WG, Higgs DR, Iborra FJ, Buckle VJ (2006) Coregulated human globin genes are frequently in spatial proximity when active. J Cell Biol 172:177-187

3. Brown JM, Green J, das Neves RP et al (2008) Association between active genes occurs at nuclear speckles and is modulated by chromatin environment. J Cell Biol 182:1083-1097

4. Schoenfelder S, Sexton T, Chakalova L et al (2010) Preferential associations between co-regulated genes reveal a transcriptional interactome in erythroid cells. Nat Genet 42:53-61

5. Cohen BA, Mitra RD, Hughes JD, Church GM (2000) A computational analysis of whole-genome expression data reveals chromosomal domains of gene expression. Nat Genet 26:183-186

6. Roy PJ, Stuart JM, Lund J, Kim SK (2002) Chromosomal clustering of muscle-expressed genes in Caenorhabditis elegans. Nature 418:975-979

7. Kosak ST, Scalzo D, Alworth SV, Li F, Palmer S, Enver T, Lee JSJ, Groudine M (2007) Coordinate gene regulation during hematopoiesis is related to genomic organization. PLoS Biol 5:e309

8. Boutanaev AM, Kalmykova AI, Shevelyov YY, Nurminsky DI (2002) Large clusters of co-expressed genes in the Drosophila genome. Nature 420:666-669

9. Mezey J, Nuzhdin S, Ye F, Jones C (2008) Coordinated evolution of co-expressed gene clusters in the Drosophila transcriptome. BMC Evol Biol 8:2

10. Purmann A, Toedling J, Schueler M, Carninci P, Lehrach H, Hayashizaki Y, Huber W, Sperling S (2007) Genomic organization of transcriptomes in mammals: coregulation and cofunctionality. Genomics 89:580-587
11. Vogel JH, von Heydebreck A, Purmann A, Sperling S (2005) Chromosomal clustering of a human transcriptome reveals regulatory background. BMC Bioinformatics 6:230

12. Nora EP, Lajoie BR, Schulz EG et al (2012) Spatial partitioning of the regulatory landscape of the $\mathrm{X}$-inactivation centre. Nature 485:381-385

13. Dixon JR, Selvaraj S, Yue F, Kim A, Li Y, Shen Y, Hu M, Liu JS, Ren B (2012) Topological domains in mammalian genomes identified by analysis of chromatin interactions. Nature 485:376-380

14. Sexton T, Yaffe E, Kenigsberg E, Bantignies F, Leblanc B, Hoichman M, Parrinello H, Tanay A, Cavalli G (2012) Three-dimensional folding and functional organization principles of the Drosophila genome. Cell 148:458-472

15. Osborne CS, Chakalova L, Mitchell JA, Horton A, Wood AL, Bolland DJ, Corcoran AE, Fraser P (2007) Myc dynamically and preferentially relocates to a transcription factory occupied by Igh. PLoS Biol 5:e192

16. Spilianakis CG, Lalioti MD, Town T, Lee GR, Flavell RA (2005) Interchromosomal associations between alternatively expressed loci. Nature 435:637-645

17. Takizawa T, Gudla PR, Guo L, Lockett S, Misteli T (2008) Allelespecific nuclear positioning of the monoallelically expressed astrocyte marker GFAP. Genes Dev 22:489-498

18. Simonis M, Klous P, Splinter E, Moshkin Y, Willemsen R, de Wit E, van Steensel B, de Laat W (2006) Nuclear organization of active and inactive chromatin domains uncovered by chromosome conformation capture-on-chip (4C). Nat Genet 38:1348-1354

19. Palstra R-J, Simonis M, Klous P, Brasset E, Eijkelkamp B, de Laat W (2008) Maintenance of long-range DNA interactions after inhibition of ongoing RNA polymerase II transcription. PLoS One 3:e1661

20. Fullwood MJ, Liu MH, Pan YF et al (2009) An oestrogen-receptor-alpha-bound human chromatin interactome. Nature 462:58-64

21. Li G, Ruan X, Auerbach RK et al (2012) Extensive promotercentered chromatin interactions provide a topological basis for transcription regulation. Cell 148:84-98

22. Shopland LS, Johnson CV, Byron M, McNeil J, Lawrence JB (2003) Clustering of multiple specific genes and gene-rich R-bands around SC-35 domains: evidence for local euchromatic neighborhoods. J Cell Biol 162:981-990

23. Szczerbal I, Bridger JM (2010) Association of adipogenic genes with SC-35 domains during porcine adipogenesis. Chromosome Res 18:887-895

24. Cook PR (1999) The organization of replication and transcription. Science 284:1790-1795

25. Iborra FJ, Pombo A, Jackson DA, Cook PR (1996) Active RNA polymerases are localized within discrete transcription "factories" in human nuclei. J Cell Sci 109(Pt 6):1427-1436

26. Iborra FJ, Pombo A, McManus J, Jackson DA, Cook PR (1996) The topology of transcription by immobilized polymerases. Exp Cell Res 229:167-173

27. Papantonis A, Larkin JD, Wada Y, Ohta Y, Ihara S, Kodama T, Cook PR (2010) Active RNA polymerases: mobile or immobile molecular machines? PLoS Biol 8:e1000419

28. Rieder D, Trajanoski Z, McNally JG (2012) Transcription factories. Front Genet 3:221

29. Cremer M, von Hase J, Volm T, Brero A, Kreth G, Walter J, Fischer C, Solovei I, Cremer C, Cremer T (2001) Non-random radial higher-order chromatin arrangements in nuclei of diploid human cells. Chromosome Res 9:541-567

30. Parada LA, McQueen PG, Munson PJ, Misteli T (2002) Conservation of relative chromosome positioning in normal and cancer cells. Curr Biol 12:1692-1697

31. Meaburn KJ, Misteli T (2008) Locus-specific and activity-independent gene repositioning during early tumorigenesis. J Cell Biol 180:39-50 
32. Rajapakse I, Perlman MD, Scalzo D, Kooperberg C, Groudine M, Kosak ST (2009) The emergence of lineage-specific chromosomal topologies from coordinate gene regulation. Proc Natl Acad Sci USA 106:6679-6684

33. Rodriguez A-M, Pisani D, Dechesne CA et al (2005) Transplantation of a multipotent cell population from human adipose tissue induces dystrophin expression in the immunocompetent $\mathrm{mdx}$ mouse. J Exp Med 201:1397-1405

34. Scheideler M, Elabd C, Zaragosi L-E et al (2008) Comparative transcriptomics of human multipotent stem cells during adipogenesis and osteoblastogenesis. BMC Genomics 9:340

35. Lamond AI, Spector DL (2003) Nuclear speckles: a model for nuclear organelles. Nat Rev Mol Cell Biol 4:605-612

36. Bond CS, Fox AH (2009) Paraspeckles: nuclear bodies built on long noncoding RNA. J Cell Biol 186:637-644

37. Ching RW, Dellaire G, Eskiw CH, Bazett-Jones DP (2005) PML bodies: a meeting place for genomic loci? J Cell Sci 118:847-854

38. Dundr M, Misteli T (2010) Biogenesis of nuclear bodies. Cold Spring Harb Perspect Biol 2:a000711

39. Chodosh LA, Fire A, Samuels M, Sharp PA (1989) 5,6-Dichloro1-beta-D-ribofuranosylbenzimidazole inhibits transcription elongation by RNA polymerase II in vitro. J Biol Chem 264:2250-2257

40. Marshall NF, Price DH (1992) Control of formation of two distinct classes of RNA polymerase II elongation complexes. Mol Cell Biol 12:2078-2090

41. Bensaude O (2011) Inhibiting eukaryotic transcription: which compound to choose? How to evaluate its activity? Transcription 2:103-108

42. Branco MR, Pombo A (2006) Intermingling of chromosome territories in interphase suggests role in translocations and transcription-dependent associations. PLoS Biol 4:e138

43. Lieberman-Aiden E, van Berkum NL, Williams L et al (2009) Comprehensive mapping of long-range interactions reveals folding principles of the human genome. Science 326:289-293

44. Olivares-Chauvet P, Fennessy D, Jackson DA, Maya-Mendoza A (2011) Innate structure of DNA foci restricts the mixing of DNA from different chromosome territories. PLoS One 6:e27527

45. Duncan PI, Stojdl DF, Marius RM, Scheit KH, Bell JC (1998) The Clk2 and Clk3 dual-specificity protein kinases regulate the intranuclear distribution of SR proteins and influence pre-mRNA splicing. Exp Cell Res 241:300-308

46. Sacco-Bubulya P, Spector DL (2002) Disassembly of interchromatin granule clusters alters the coordination of transcription and pre-mRNA splicing. J Cell Biol 156:425-436

47. Tilgner H, Knowles DG, Johnson R, Davis CA, Chakrabortty S, Djebali S, Curado J, Snyder M, Gingeras TR, Guigo R (2012) Deep sequencing of subcellular RNA fractions shows splicing to be predominantly co-transcriptional in the human genome but inefficient for lncRNAs. Genome Res 22:1616-1625

48. Hepperger C, Mannes A, Merz J, Peters J, Dietzel S (2008) Three-dimensional positioning of genes in mouse cell nuclei. Chromosoma 117:535-551

49. Murmann AE, Gao J, Encinosa M, Gautier M, Peter ME, Eils R, Lichter P, Rowley JD (2005) Local gene density predicts the spatial position of genetic loci in the interphase nucleus. Exp Cell Res 311:14-26

50. Küpper K, Kölbl A, Biener D et al (2007) Radial chromatin positioning is shaped by local gene density, not by gene expression. Chromosoma 116:285-306

51. Chubb JR, Boyle S, Perry P, Bickmore WA (2002) Chromatin motion is constrained by association with nuclear compartments in human cells. Curr Biol 12:439-445

52. Hakim O, Sung M-H, Voss TC, Splinter E, John S, Sabo PJ, Thurman RE, Stamatoyannopoulos JA, de Laat W, Hager GL (2011)
Diverse gene reprogramming events occur in the same spatial clusters of distal regulatory elements. Genome Res 21:697-706

53. Lin S, Coutinho-Mansfield G, Wang D, Pandit S, Fu X-D (2008) The splicing factor SC35 has an active role in transcriptional elongation. Nat Struct Mol Biol 15:819-826

54. Eskiw CH, Rapp A, Carter DRF, Cook PR (2008) RNA polymerase II activity is located on the surface of protein-rich transcription factories. J Cell Sci 121:1999-2007

55. Eskiw CH, Fraser P (2011) Ultrastructural study of transcription factories in mouse erythroblasts. J Cell Sci 124:3676-3683

56. Ghamari A, van de Corput MPC, Thongjuea S, van Cappellen WA, van Ijcken W, van Haren J, Soler E, Eick D, Lenhard B, Grosveld FG (2013) In vivo live imaging of RNA polymerase II transcription factories in primary cells. Genes Dev 27:767-777

57. Ferrai C, Xie SQ, Luraghi P, Munari D, Ramirez F, Branco MR, Pombo A, Crippa MP (2010) Poised transcription factories prime silent uPA gene prior to activation. PLoS Biol 8:e1000270

58. Mitchell JA, Fraser P (2008) Transcription factories are nuclear subcompartments that remain in the absence of transcription. Genes Dev 22:20-25

59. Hakim O, Resch W, Yamane A et al (2012) DNA damage defines sites of recurrent chromosomal translocations in B lymphocytes. Nature 484:69-74

60. Ploner C, Rainer J, Niederegger H, Eduardoff M, Villunger A, Geley S, Kofler R (2008) The BCL2 rheostat in glucocorticoidinduced apoptosis of acute lymphoblastic leukemia. Leukemia 22:370-377

61. Hepperger C, Otten S, von Hase J, Dietzel S (2007) Preservation of large-scale chromatin structure in FISH experiments. Chromosoma 116:117-133

62. Solovei I, Cavallo A, Schermelleh L, Jaunin F, Scasselati C, Cmarko D, Cremer C, Fakan S, Cremer T (2002) Spatial preservation of nuclear chromatin architecture during three-dimensional fluorescence in situ hybridization (3D-FISH). Exp Cell Res 276:10-23

63. Markaki Y, Smeets D, Fiedler S, Schmid VJ, Schermelleh L, Cremer T, Cremer M (2012) The potential of 3D-FISH and superresolution structured illumination microscopy for studies of 3D nuclear architecture: 3D structured illumination microscopy of defined chromosomal structures visualized by 3D (immuno)FISH opens new perspectives for studies of nuclear architecture. BioEssays 34:412-426

64. Müller WG, Rieder D, Kreth G, Cremer C, Trajanoski Z, McNally JG (2004) Generic features of tertiary chromatin structure as detected in natural chromosomes. Mol Cell Biol 24:9359-9370

65. Telenius H, Pelmear AH, Tunnacliffe A, Carter NP, Behmel A, Ferguson-Smith MA, Nordenskjöld M, Pfragner R, Ponder BA (1992) Cytogenetic analysis by chromosome painting using DOP-PCR amplified flow-sorted chromosomes. Genes Chromosom Cancer 4:257-263

66. Solovei I, Walter J, Cremer M, Habermann F, Schermelleh L, Cremer T (2002) FISH, a practical approach. In: Beatty B, Mai S, Squire J (eds) Oxford University Press, pp 119-157

67. Müller WG, Rieder D, Karpova TS, John S, Trajanoski Z, McNally JG (2007) Organization of chromatin and histone modifications at a transcription site. J Cell Biol 177:957-967

68. Bright Wilson EJR (1952) An introduction to scientific research. Dover Publications, Mineola, NY

69. McManus KJ, Stephens DA, Adams NM, Islam SA, Freemont PS, Hendzel MJ (2006) The transcriptional regulator CBP has defined spatial associations within interphase nuclei. PLoS Comput Biol 2:e139 